\title{
Coupling continuum dislocation transport with crystal plasticity for application to shock loading conditions
}

\author{
D.J. Luscher ${ }^{\mathrm{a}}$, J.R. Mayeur ${ }^{\mathrm{a}}$, H.M. Mourad ${ }^{\mathrm{a}}$, A. Hunter ${ }^{\mathrm{b}}$, M.A. Kenamond ${ }^{\mathrm{b}}$ \\ ${ }^{a}$ Theoretical Division, Fluid Dynamics and Solid Mechanics Group \\ ${ }^{b}$ Computational Physics Division, Lagrangian Codes Group \\ Los Alamos National Laboratory, Los Alamos, NM
}

\begin{abstract}
We have developed a multi-physics modeling approach that couples continuum dislocation transport, nonlinear thermoelasticity, crystal plasticity, and consistent internal stress and deformation fields to simulate the single-crystal response of materials under extreme dynamic conditions. Dislocation transport is modeled by enforcing dislocation conservation at a slip-system level through the solution of advection-diffusion equations. Nonlinear thermoelasticity provides a thermodynamically consistent equation of state to relate stress (including pressure), temperature, energy densities, and dissipation. Crystal plasticity is coupled to dislocation transport via Orowan's expression where the constitutive description makes use of recent advances in dislocation velocity theories applicable under extreme loading conditions. The configuration of geometrically necessary dislocation density gives rise to an internal stress field that can either inhibit or accentuate the flow of dislocations. An internal strain field associated with the internal stress field contributes to the kinematic decomposition of the overall deformation. The paper describes each theoretical component of the framework, key aspects of the constitutive theory, and some details of a one-dimensional implementation. Results from single-crystal copper plate impact simulations are discussed in order to highlight the role of dislocation transport and pile-up in shock loading regimes. The main conclusions of the paper reinforce the utility of the modeling approach to shock problems.
\end{abstract}

Keywords: crystal plasticity, shock waves, dislocations, dynamics

\section{Introduction}

An improved mechanistic understanding of the mesoscale response of materials under extreme dynamic loading conditions is required to enable predictive physics-based modeling of macroscale response under such conditions. These environmental extremes often include large pressures, deformations, and deformation rates. The response of materials to such environments involves complex physical processes, for example, the nucleation of voids and their subsequent growth and interaction, nucleation of twins, phase changes, and the formation of shear bands. Many of these physical processes occur over length and time scales that are prohibitively small to explicitly resolve within macroscale calculations.

Mesoscale modeling and simulation can provide a physical connection between important microstructural processes (e.g. plasticity, damage nucleation, phase transformation, twinning) occurring under environmental extremes and macroscale models of engineered performance. In the case of polycrystalline materials, this connection can be established via theoretical and numerical mesoscale models of polycrystal response, only if such models include accurate descriptions of the underlying single crystal physics. In addition, mesoscale simulation results can assist in the interpretation of experimental observations in many cases. Together, mesoscale modeling and experiments with high-resolution diagnostics can inform the development of improved macroscale damage and plasticity models.

For example, plate impact experiments have been conducted to gain a better understanding of the conditions leading to nucleation of voids in single and polycrystalline copper (Cao et al., 2010; Perez-Bergquist et al., 2011; Cerreta et al., 2012; Han et al., 2012). These experimental results can be used to formulate 
hypotheses for and/or lend credibility to kinetic models of void nucleation in macroscale porosity-dependent plasticity. In particular, this previous experimental work has established correlations between grain boundary misorientation and void nucleation clearly indicating that certain special grain boundary types are naturally more resistant to void nucleation. However, neither the specific details of local material state at incipient nucleation nor the exact chronology of the process can be ascertained directly from these experiments.

Under favorable conditions, plastic deformation in metals is accommodated at the microscale by slip on discrete crystallographic planes via the motion of dislocations through the atomic lattice. A prescribed deformation can be accommodated by crystallographic slip, provided a sufficient population of mobile dislocations is available. In contrast, under extreme loading conditions, there may be an insufficient number of freely mobile dislocations, or those available may be unable to move through the lattice at sufficient velocity to accommodate the imposed deformation. If plastic slip alone cannot account for the imposed deformation over the relevant length and time scales, additional dislocations must be nucleated or alternative deformation mechanisms (e.g. twinning, damage) must be activated. Thus, developing an understanding of the interplay of these various competing mechanisms requires a modeling capability that includes a detailed representation of plastic slip kinetics.

Early crystal plasticity models employed phenomenological expressions for the plastic slip kinetics and hardening relations and did not include an explicit representation of dislocations (e.g. Asaro, 1983; Mathur and Dawson, 1989; Kalidindi et al., 1992; Bronkhorst et al., 1992). Subsequent improvements introduced dislocation density as an evolving local state variable, rather than a continuum field variable (e.g. Roters et al., 2000; Prasad et al., 2005; Ma et al., 2006; Lee et al., 2010; Alankar et al., 2011; Lim et al., 2011). Such theories considered the dislocation density, at any material point, to evolve independently of the material state, history, and evolutionary processes at neighboring points. Nonlocal dislocation-dislocation interactions, which can have a significant effect on slip in some cases, were ignored in these approaches. Therefore, the resulting models did not capture the effect of dislocation accumulation, e.g. a pile-up at a grain boundary, lacked an inherent length scale, and consequently, were unable to predict size effects, e.g. Hall-Petch strengthening.

A number of nonlocal crystal plasticity models, reflecting dislocation-dislocation interactions with varying levels of fidelity, were developed to address the aforementioned limitations. Many prevalent nonlocal models are based on higher-order generalized continuum field theories, which include micromorphic (Forest et al., 1997; Bammann, 2001; Forest, 2009; Aslan et al., 2011; Mayeur et al., 2011), and slip gradient (Gurtin, 2002; Yefimov et al., 2004; Han et al., 2005; Gurtin et al., 2007; Gerken and Dawson, 2008; Bayley et al., 2006) approaches. One of the main shortcomings of this class of models is that, while many of them are based on a direct kinematic connection with an underlying conservation principle for the geometrically necessary content of a dislocation field (GND) and its relation with the evolution of Nye's tensor, they do not impose any conservation requirement on the remainder of the dislocation field, often referred to as statistically stored dislocation (SSD) field. Thus, a proper conservation of the total dislocation density and the consequences it has on the evolution of slip are not properly captured in such models.

Arsenlis and Parks (1999) outline relationships between the geometrically necessary and statistically stored components of a dislocation field and Nye's tensor initially established by Nye (1953), Kröner (1961), and Ashby (1970) to connect dislocation mechanics with strain gradient theories. Arsenlis and Parks (1999) suggested that, because of the known kinematic properties of dislocations, the most rigorous framework for modeling dislocation density would be to construct and solve an appropriate initial boundary value problem from which Nye's tensor can be computed directly. Dislocation density conservation equations were derived through application of statistical mechanics averaging concepts to systems of discrete dislocations (Groma et al., 2003). This approach has early precedent set by Bammann and Aifantis (1982) and Walgraef and Aifantis (1985a,b,c) who developed a generalized set of partial differential equations (PDEs) reflecting the conservation of mass and momentum for diffusive species. Yefimov and van der Giessen (2005a) recast this approach by deriving conservation equations for total, $\varrho$, and geometrically necessary, $\kappa$, parts of dislocation density for multiple slip systems, which was subsequently employed by Limkumnerd and van der Giessen (2008b,a). Hirschberger et al. (2011) compare the performance of continuum dislocation flux theories with slip-gradient theories and outline conditions under which the latter provide reasonable approximations to 
the former. Hochrainer et al. (2007) and Sandfeld et al. (2010,2011) have developed a continuum dislocation dynamics theory that includes a higher-resolution description of the dislocation field. In particular, variants of the theory include degrees-of-freedom associated with dislocation line direction and curvature. Their approach enables the specification of detailed constitutive relationships governing the kinetic interactions of dislocations, but is computationally demanding.

More recently, Reuber et al. (2014) present a comprehensive theory of continuum dislocation transport that tracks positive and negative edge and screw dislocation densities that is more tractable from a computational perspective. They compare results from simulations of quasi-static wedge indentation of single crystal nickel with measurements of lattice rotation and GND distribution from experiments of Kysar et al. (2010). Their constitutive description includes detailed expressions for dislocation interactions including forest hardening, dislocation multiplication, and dipole creation and annihilation.

An interesting alternative framework that accounts for dislocation transport and conservation has been developed by Acharya and co-workers (Acharya, 2001, 2003; Acharya and Roy, 2006; Acharya, 2007). This model employs a Stokes-Helmholtz decomposition of the elastic (and plastic) deformation map(s) used in classical finite deformation crystal plasticity. A tensorial balance equation is derived for Nye's tensor and its evolution is directly linked to the evolution of the incompatible part, i.e. the part with non-vanishing curl, of the inverse elastic deformation map. With the aid of a constitutive equation for the compatible part of the elastic deformation gradient, the incompatible part is obtained by solving an additional initial-boundary value problem. A noteworthy feature of this modeling framework is that it can exactly capture the stress fields of individual dislocations.

Several researchers pursuing nonlocal continuum theories of crystal plasticity use a plane strain solution assuming isotropic elasticity for the stress field surrounding the Volterra dislocation to compute either the internal stress field or a slip-system associated back stress (Evers et al., 2004a,b; Yefimov and van der Giessen, 2005a; Bayley et al., 2006; Geers et al., 2007; Gerken and Dawson, 2008; Hirschberger et al., 2011; Liu et al., 2011; Leung et al., 2015; Schulz et al., 2014). Leung et al. (2015), incorporate a physical representation of the long-range internal stress by generalizing relations for the elastic interaction force between two dislocations from Hirth and Lothe (1982). While they discuss the long-range kinematic effects associated with this physical long-range stress field, they do not directly incorporate it into their solution. Gerken and Dawson (2008) directly address the kinematic significance of a configuration of GNDs through the three term multiplicative decomposition of the deformation gradient introduced previously by Hartley (2003), Clayton and McDowell (2003), and Clayton et al. (2004). Within a small strain geometrical setting, Aghababaei et al. (2011) use a kinematic decomposition similar to Acharya (2001) and compute the external and long-range internal stress fields by obtaining a Green's function solution to the Beltrami stress function adapted to the incompatible part of elastic distortion.

All of the aforementioned models that couple continuum dislocation transport with crystal plasticity are directed at quasi-static or relatively low rate dynamic response. A handful of recent works have focused on extending crystal plasticity models for application to shock conditions (Schoenfeld, 1998; Becker, 2004; Winey and Gupta, 2004; Barton et al., 2005; Winey and Gupta, 2006; Vogler and Clayton, 2008; Barton et al., 2008; Lloyd et al., 2014; Clayton, 2014), although they all employ a local description of crystal plasticity.

To put the above ideas in context and motivate the proposed work, we use the example of dislocation pile-up at a grain boundary (GB). Such a pile-up may eventually drive dislocation penetration (i.e. slip transmission) through the GB or source activation on the other side of the GB. Conversely, the resistance to slip transmission may initiate an alternative mechanism, such as damage nucleation, to accommodate the prescribed deformation. In fact, porosity nucleation is known to occur at GBs in metals under shock loading conditions. And while it is experimentally observed that specific microstructural details (e.g. crystallographic orientations, GB character, deformation rate) affect this process (Perez-Bergquist et al., 2011; Cerreta et al., 2012), accurate representations of the relevant deformation mechanisms under these conditions are beyond the reach of existing mesoscale modeling approaches.

A predictive modeling capability for phenomena occurring at material interfaces during shock loading of solids demands a proper, physics-based treatment of the fundamentally nonlocal interactions involving dislocations and interfaces. Our modeling approach is outlined in Figure 1 which makes use of the nomen- 
clature defined by Table 1. Crystal plasticity is modeled as a coupled initial- and boundary-value problem (IBVP), consisting of three sub-problems: deformation momentum balance (DMB), continuum dislocation transport (CDT), and dislocation-deformation compatibility (DDC). The DMB sub-problem reflects classical continuum deformation field theory, with the displacement field as the primary unknown variable. This sub-problem is governed by the principle of conservation of momentum under the applied loading. The CDT sub-problem is governed by conservation laws for the densities of dislocation populations which are treated as primary field variables. The DDC sub-problem reconciles evolving dislocation fields from CDT with the deformation and stress fields of DMB. Specifically, solving the DDC sub-problem is necessary to identify the long-range residual stress field, and associated strain field, attributed to the distribution of non-redundant dislocations in the lattice. The coupling between these sub-problems reflects (i) that plastic deformation (DMB) is accommodated by dislocation motion (CDT), (ii) the redistribution of dislocations (CDT) within a crystalline material is driven by stress (DMB, DDC), and (iii) a net polarity in the dislocation density fields (CDT) gives rise to long-range internal, residual stresses (DDC).

It is noted that the general approach depicted in Figure 1 encompasses numerous specific formulations. These include not only the theory and implementation presented here, but also models in the recent literature, e.g. (Yefimov and van der Giessen, 2005a; Gerken and Dawson, 2008; Hirschberger et al., 2011; Reuber et al., 2014), which incorporate a subset of - or similar approaches to - the features depicted in Figure 1. The novelty of our approach is built upon explicit representation of dislocation transport (CDT), detailed accounting for elastic interactions involving geometrically necessary dislocations (DDC), classical momentum balance (DMB), and the tight coupling between all three of these sub-problems, in balance with constitutive theories that are germane to the high strain-rate and large elastic and plastic deformation regimes.

The remainder of this paper is organized as follows. Section 2 provides theoretical details regarding the deformation-momentum balance sub-problem. In particular, this section comprises governing equations, constitutive equations for dislocation velocity and slip rates, and nonlinear thermoelasticity. Section 3 addresses the continuum dislocation transport sub-problem including governing equations and constitutive descriptions for dislocation interactions. The dislocation-deformation compatibility sub-problem is addressed in Section 4. Aspects of the 1D implementation pursued here are briefly addressed in Section 5 along with numerical simulations of plate impact scenarios. Summarizing conclusions highlighting the results of this work and anticipated future contributions in this area are provided in Section 6.

In the text, scalar variables are denoted with italic font, while tensoral variables of order one and two are denoted by bold type, e.g. A. Fourth order tensors are indicated by blackboard font, i.e., $\mathbb{C}$. Coefficients of tensors resolved against Cartesian base vectors $\mathbf{e}_{i}$ are expressed using subscripts, $\mathbf{A}=A_{i j} \mathbf{e}_{i} \otimes \mathbf{e}_{j}$ and the operator $\otimes$ denotes the dyadic outer product of tensors. The transpose of a tensor is indicated by a superscript ' $T$ ' as in $\mathbf{A}^{T}$. As indicated in Table 1, the operator ' $:$ ' is used to indicate double contraction of tensors, e.g. $\mathbf{A}=\mathbb{C}: \mathbf{B}$ corresponds to $A_{i j} \mathbf{e}_{i} \otimes \mathbf{e}_{j}=\mathbb{C}_{i j k l} B_{k l} \mathbf{e}_{i} \otimes \mathbf{e}_{j}$ where standard indicial notation and summation convention are used except if indicated otherwise. Appearance of tensors adjacent to each other with no operator implies an inner product or contraction as in, $\mathbf{F x}=F_{i j} x_{j} \mathbf{e}_{i}$ or $\mathbf{F A}=F_{i k} A_{k j} \mathbf{e}_{i} \otimes \mathbf{e}_{j}$. In some cases, for clarity, the scalar product of vectors or gradient operators is denoted by $\mathbf{v} \cdot \mathbf{x}$ or $\nabla \cdot \mathbf{v}$, for example.

\section{Deformation Momentum Balance}

\section{Governing Equations}

The primary field variable of the DMB sub-problem is the deformed position of material points, i.e., $\mathbf{x}=\Phi(t, \mathbf{X})$, where $\mathbf{X}$ is a vector defining the initial position of a material point, $t$ is time, and $\Phi$ maps initial positions to deformed positions throughout time. The strong form of conservation of linear momentum is expressed in its Lagrangian form as

$$
\nabla_{\mathrm{o}} \cdot \mathbf{P}=\bar{\rho}_{\mathrm{o}} \ddot{\mathbf{x}}
$$

where $\mathbf{P}$ is the nominal stress (transpose of first Piola-Kirchhoff stress), $\bar{\rho}_{\mathrm{o}}$ is the mass density per unit undeformed volume, and $\nabla_{\mathrm{o}}$ is the gradient operator with respect to the undeformed configuration. The 
Table 1: Definitions of variables listed in this table are provided to clarify Figure 1. Only the most significant variables are defined; all variables and parameters are defined either here or where they are first introduced.

\begin{tabular}{|c|c|}
\hline Var & Definition \\
\hline$\alpha, \beta$ & Indices used to represent $\alpha^{t h}$ and $\beta^{\text {th }}$ slip systems \\
\hline $\mathbf{b}^{\alpha}$ & Burger's vector of slip system $\alpha$ \\
\hline$b$ & Nominal magnitude of Burger's vector \\
\hline $\mathbb{C}$ & 4th order elasticity tensor (generally anisotropic) \\
\hline $\mathbf{E}_{\mathrm{e}}$ & Elastic Green-Lagrange (GL) strain tensor \\
\hline$\epsilon_{\mathrm{i}}$ & Internal small strain tensor associated with dislocation field \\
\hline $\mathbf{F}$ & Deformation gradient tensor $\left(\mathbf{F}=\nabla_{\mathrm{o}} \mathbf{x}\right)$ \\
\hline $\mathbf{F}_{\mathrm{e}}$ & Elastic part of deformation gradient tensor \\
\hline $\mathbf{F}_{\mathrm{i}}$ & Part of deformation gradient associated with internal stress field \\
\hline $\mathbf{F}_{\mathrm{p}}$ & Plastic part of deformation gradient tensor \\
\hline$\kappa^{\alpha}$ & Net dislocation density considering polarity (GN density) \\
\hline$\mu$ & Nominal elastic shear modulus \\
\hline $\mathbf{n}_{\mathrm{o}}^{\alpha}, \mathbf{n}^{\alpha}$ & Slip plane normal vector in reference, current configuration \\
\hline$N_{\mathrm{s}}$ & Number of slip systems \\
\hline $\mathbf{P}$ & Nominal stress (transpose of 1st Piola-Kirchhoff) \\
\hline$\psi$ & Helmholtz free energy \\
\hline $\bar{\rho}_{\mathrm{o}}$ & Specific mass per unit volume in reference configuration \\
\hline$\varrho^{\alpha}$ & Total (or gross) dislocation line length per unit volume (i.e. density) \\
\hline$\varrho_{\perp}^{\alpha}$ & Density of dislocations of edge character with a positive sense \\
\hline$\varrho^{\alpha} \bar{\alpha}$ & Density of dislocations of edge character with a negative sense \\
\hline$s^{\alpha}$ & Dislocation source term (e.g. multiplication, annihilation) \\
\hline${\underset{\sim}{\mathrm{O}}}_{\mathrm{O}}^{\alpha}, \mathbf{s}^{\alpha}$ & Slip direction vectors in reference, current configuration \\
\hline$\widetilde{\mathbf{S}}$ & 2nd Piola-Kirchhoff stress with respect to intermediate configuration \\
\hline $\mathbf{S}_{\mathrm{i}}$ & Internal 2nd Piola-Kirchhoff stress associated with dislocation field \\
\hline$\sigma$ & External Cauchy stress field \\
\hline$\sigma_{\mathrm{i}}$ & Long range internal Cauchy stress field \\
\hline$T, \Delta T$ & Temperature, change in temperature from a reference state \\
\hline$\tau_{\mathrm{i}}^{\alpha}$ & Internal stress along a slip system due to GND distribution on that system \\
\hline $\mathbf{v}^{\alpha}$ & Dislocation velocity vector \\
\hline $\mathbf{x}, \dot{\mathbf{x}}, \ddot{\mathbf{x}}$ & Position, velocity, and acceleration of material point in current conf. \\
\hline Operator/annotation & Definition \\
\hline$\nabla_{\mathrm{o}}$ & Gradient operator with respect to fixed reference material coordinates \\
\hline$\dot{c}$ & Time rate of change of variable $c$ \\
\hline$\hat{c}$ & Prescribed constitutive function for the evaluation of variable $c$ \\
\hline$\times$ & Cross product \\
\hline$\otimes$ & Dyadic outer product \\
\hline & Mixed outer product, i.e., $\mathbb{C}=\mathbf{A} \otimes \mathbf{B}$ corresponds to $C_{i j k l}=A_{i k} B_{j l}$ \\
\hline $\mathbf{A}: \mathbf{B}$ & Product $A_{i j} B_{i j}$ \\
\hline
\end{tabular}




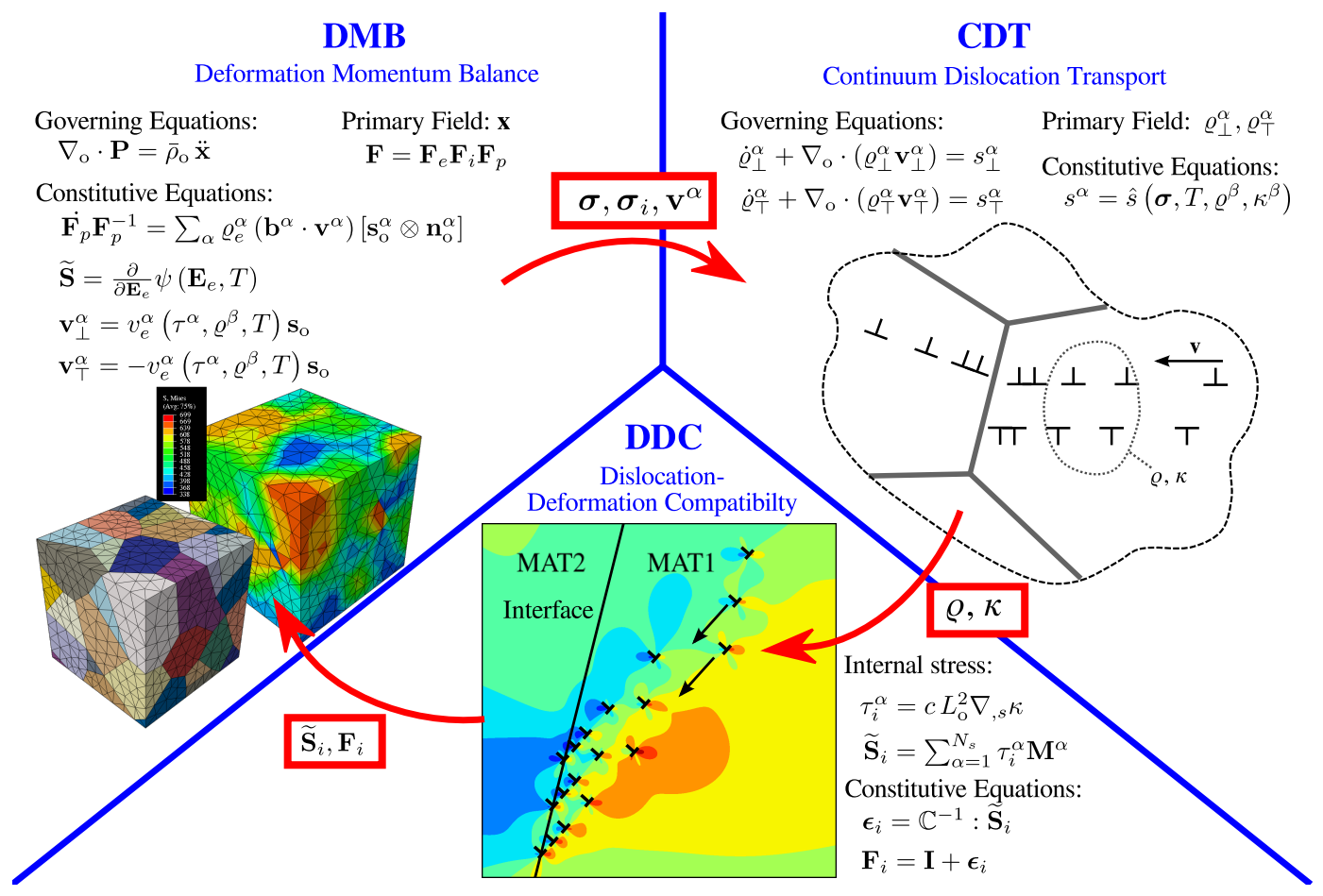

Figure 1: Illustration of the coupled multi-physics approach for dislocation-based crystal plasticity. The coupled problem is decomposed into three sub-problems, viz. Deformation Momentum Balance (DMB), Continuum Dislocation Transport (CDT), and Dislocation Deformation Compatibility (DDC). Red arrows indicate coupling between sub-problems via the flow of field variables. Variables and operators are defined in Table 1 as well as within the text. 
strong form of angular momentum conservation is typically satisfied through constitutive behavior and can be expressed as $\mathbf{F} \cdot \mathbf{P}=\mathbf{P}^{T} \cdot \mathbf{F}^{T}$ where the deformation gradient is defined as $\mathbf{F}=\mathbf{x} \nabla_{\mathrm{o}}$. In this work we focus on shock propagation, for example, during a plate impact experiment, and specifically address the case of $1 \mathrm{D}$ deformation described as follows. The displacement field is generally three dimensional, but varies only along a 1D coordinate axis, i.e.,

$$
\mathbf{x}=\mathbf{x}\left(X_{1}\right)
$$

consequently, conservation of linear momentum from Eq. (1) is reduced to

$$
\frac{\partial P_{1 i}}{\partial X_{1}}-\bar{\rho}_{\mathrm{o}} \ddot{x}_{i}=0
$$

\section{Kinematics}

Following the arguments of Hartley (2003), Clayton and McDowell (2003), and Clayton et al. (2004), the deformation gradient is decomposed into a thermoelastic part $\mathbf{F}_{\mathrm{e}}$, a part that represents the cumulative effects of crystallographic slip, $\mathbf{F}_{\mathrm{p}}$, and a component due to the elastic incompatibility accounting for the presence of dislocations within the lattice, $\mathbf{F}_{\mathrm{i}}$, according to

$$
\mathbf{F}=\mathbf{F}_{\mathrm{e}} \mathbf{F}_{\mathrm{i}} \mathbf{F}_{\mathrm{p}}
$$

The elastic Cauchy deformation and elastic Green-Lagrange strain tensors are computed from the elastic part of the deformation gradient by $\mathbf{C}_{\mathrm{e}}=\mathbf{F}_{\mathrm{e}}^{T} \mathbf{F}_{\mathrm{e}}$ and $\mathbf{E}_{\mathrm{e}}=\frac{1}{2}\left(\mathbf{C}_{\mathrm{e}}-\mathbf{I}\right)$, respectively. For the case of cubic material symmetry we use the decomposition of Luscher et al. (2013) to decouple the volumetric equation of state from the isochoric deformation and associated elasto-plasticity. Thus, we define the isochoric elastic deformation gradient

$$
\overline{\mathbf{F}}_{\mathrm{e}}=J_{\mathrm{e}}^{-1 / 3} \mathbf{F}_{\mathrm{e}}
$$

where $J_{\mathrm{e}}=\operatorname{det} \mathbf{F}_{\mathrm{e}}$. The corresponding isochoric elastic Cauchy deformation and Green strain are defined as $\overline{\mathbf{C}}_{\mathrm{e}}=\overline{\mathbf{F}}_{\mathrm{e}}^{T} \overline{\mathbf{F}}_{\mathrm{e}}$ and $\overline{\mathbf{E}}_{\mathrm{e}}=\frac{1}{2}\left(\overline{\mathbf{C}}_{\mathrm{e}}-\mathbf{I}\right)$, respectively, and reflect purely isochoric deformation. Presently, we restrict attention to the case where $\operatorname{det} \mathbf{F}_{\mathrm{i}} \mathbf{F}_{\mathrm{p}}=1$ such that $J=\operatorname{det} \mathbf{F}=J_{\mathrm{e}}$ and the specific volume is obtained via $\mathrm{v}=J / \bar{\rho}_{\mathrm{o}}$.

Recently, Clayton et al. (Clayton, 2013, 2014) have proposed an alternate elastic strain measure, $\epsilon=\frac{1}{2}\left(\mathbf{I}-\mathbf{F}_{\mathrm{e}}^{-1} \mathbf{F}_{\mathrm{e}}^{-T}\right)$. In that work, they demonstrate that their elastic strain measure results in better agreement between simulated and measured Hugoniot data when considering only quadratic or cubic freeenergy potentials to define the nonlinear thermoelastic state of the material. In such cases, the mechanical nonlinearity of thermoelastic response is solely captured through the third-order elastic constants and the nonlinear relationship between strain and deformation. The nature of their strain definition is better suited to represent this behavior for uniaxial impact problems when using an n-order polynomial to represent the strain energy density of the material. In this work, we capture the nonlinear thermoelasticity through the equation-of-state developed by Greeff et al. (2006) using the kinematic framework developed in Luscher et al. (2013). This approach has been demonstrated to adequately represent the nonlinear thermoelastic response of copper up to shock pressures of 100GPa.

\section{Thermoelasticity}

The relationship between thermoelastic stresses, deformation, and temperature, $T$, is provided by a thermodynamic equation of state for the Helmholtz free energy expressed as

$$
\psi=\hat{\psi}\left(\mathbf{E}_{\mathrm{e}}, T, \varrho_{\mathrm{c}}\right)=\hat{\psi}_{\mathrm{v}}(\mathrm{v}, T)+\hat{\psi}_{\mathrm{d}}\left(\overline{\mathbf{E}}_{\mathrm{e}}, T\right)+\hat{\psi}_{\mathrm{s}}\left(\varrho_{\mathrm{c}}\right)
$$

where $\hat{\psi}_{\mathrm{v}}(\mathrm{v}, T)$ reflects the equation of state for the pressure-volume-temperature behavior, $\hat{\psi}_{\mathrm{d}}\left(\overline{\mathbf{E}}_{\mathrm{e}}, T\right)$ is a contribution to free energy from recoverable 'deviatoric' elastic strain, and $\hat{\psi}_{\mathrm{s}}$ is free energy storage associated with the local state and nonlocal distribution of dislocation density. The second Piola-Kirchhoff stress with 
respect to the intermediate configuration is computed from the Helmholtz free energy, making use of the chain rule of differentiation, according to

$$
\widetilde{\mathbf{S}}=\frac{\partial \psi}{\partial \mathbf{E}_{\mathrm{e}}}=-p J \mathbf{C}_{\mathrm{e}}^{-1}+\frac{\partial \hat{\psi}_{\mathrm{d}}}{\partial \overline{\mathbf{E}}_{\mathrm{e}}}: \mathbb{M}
$$

where the thermodynamic pressure is defined as $p=-\frac{\partial \psi_{\mathrm{v}}}{\partial \mathrm{v}}$ and $\mathbb{M}=J^{-2 / 3}\left(\mathbf{I} \otimes \mathbf{I}-\frac{1}{3} \mathbf{C}_{\mathrm{e}} \otimes \mathbf{C}_{\mathrm{e}}^{-1}\right)(\mathrm{Luscher}$ et al., 2013). The Cauchy stress is obtained by a push forward of $\widetilde{\mathbf{S}}$ by the elastic deformation map and the nominal stress is related to the Cauchy stress through the standard relation, i.e.,

$$
\boldsymbol{\sigma}=J_{\mathrm{e}}^{-1} \mathbf{F}_{\mathrm{e}} \widetilde{\mathbf{S}} \mathbf{e}_{\mathrm{e}}^{T} \quad \text { and } \quad \mathbf{P}=J \mathbf{F}^{-1} \boldsymbol{\sigma}
$$

Greeff et al. (2006) developed an equation of state for the pressure-volume-temperature relationship of fcc $\mathrm{Cu}$ expressed as a Helmholtz free energy state function according to

$$
\hat{\psi}_{\mathrm{v}}=\hat{\psi}_{0}(\mathrm{v})+\hat{\psi}_{\text {ion }}(\mathrm{v}, T)+\hat{\psi}_{\mathrm{el}}(\mathrm{v}, T)
$$

where $\hat{\psi}_{0}$ is the static lattice energy, $\hat{\psi}_{\text {ion }}$ is the ion motion free energy, and $\hat{\psi}_{\text {el }}$ is the electronic excitation free energy. We use the model developed by Greeff et al. (2006) directly and present only their final equations and parameters here. The static lattice contribution to free energy is based on the empirical state function developed by Vinet et al. (1986), i.e.,

$$
\hat{\psi}_{0}(\mathrm{v})=\frac{4 \mathrm{v}^{*} B^{*}}{\left(B_{1}-1\right)^{2}}(1-(1+X) \exp [-X])
$$

where $X=\frac{3}{2}\left(B_{1}-1\right)\left(\left(\frac{\mathrm{v}}{\mathrm{v}^{*}}\right)^{1 / 3}-1\right)$ and $\mathrm{v}^{*}, B^{*}$, and $B_{1}$ are parameters identified to reproduce measured bulk modulus and density under ambient conditions and experimental Hugoniot data (Greeff et al., 2006). Contributions to free energy from ion motion are expressed by the Debye free energy function

$$
\hat{\psi}_{\text {ion }}(\mathrm{v}, T)=\frac{R}{M_{\text {mol }}}\left(\frac{9}{8} T_{D}+3 T \ln \left(1-\exp \left[-T_{D} / T\right]\right)-T \hat{D}\left[T_{D} / T\right]\right)
$$

where $R$ is the molar gas constant, $M_{\mathrm{mol}}$ is the molar mass, $\hat{D}$ is the Debye energy function (Girifalco, 2000) expressed in terms of $y=T_{D} / T$ as

$$
\hat{D}[y]=\frac{3}{y^{3}} \int_{0}^{y}\left(\frac{z^{3}}{e^{z}-1}\right) d z
$$

and $T_{D}$ is the effective Debye temperature expressed as a function of specific volume by

$$
T_{D}(\mathrm{v})=T_{D 0}\left(\frac{\mathrm{v}}{\mathrm{v}_{0}}\right)^{-\gamma^{\infty}} \exp \left[-a\left(\frac{\mathrm{v}}{\mathrm{v}_{0}}-1\right)-\frac{b}{2}\left(\left(\frac{\mathrm{v}}{\mathrm{v}_{0}}\right)^{2}-1\right)\right]
$$

where $T_{D 0}$ is the Debye temperature at the reference state, and $a, b$, and $\gamma^{\infty}$ are parameters from an expression for the Grüneisen coefficient. The electronic excitation free energy is given as

$$
\hat{\psi}_{\mathrm{el}}(\mathrm{v}, T)=-\frac{1}{2} \Gamma_{0} T^{2}\left(\frac{\mathrm{v}}{\mathrm{v}_{0}}\right)^{\kappa_{\mathrm{s}}}
$$

where $\Gamma_{0}$ is the Sommerfeld coefficient at the reference state and $\kappa_{\mathrm{S}}$ is a parameter reflecting the relationship between Sommerfeld coefficient and Fermi energy over a range of states (Greeff and Graf, 2004). The equation of state model parameters used here are those identified by Greeff et al. (2006) and are listed in Table 2.

The deviatoric part of the Helmholtz free energy depends upon the isochoric elastic Green Lagrange strain through the quadratic expression

$$
\hat{\psi}_{\mathrm{d}}\left(\overline{\mathbf{E}}_{\mathrm{e}}, T\right)=\frac{1}{2} \overline{\mathbf{E}}_{\mathrm{e}}: \mathbb{C}(T, p): \overline{\mathbf{E}}_{\mathrm{e}}
$$


where $\mathbb{C}$ is the fourth order elasticity tensor. In this work, we treat the coefficients of $\mathbb{C}$ as constant with values listed in Table 2, although in general our framework allows a linear temperature and pressure dependence according to

$$
C_{i j}=C_{i j}^{\mathrm{r}}+C_{i j, p} p+C_{i j, T}\left(T-T_{\mathrm{r}}\right)
$$

where $C_{i j}^{\mathrm{r}}$ are the elastic coefficients in Voigt notation corresponding to reference conditions (zero pressure and temperature $T_{\mathrm{r}}$ ) and $C_{i j, p}$ and $C_{i j, T}$ are the first derivatives of elastic constants with respect to pressure and temperature, respectively.

\section{Crystal plasticity}

We assume a field of dislocation density (line length per unit volume) with positive and negative polarities is distributed amongst $N_{\mathrm{s}}$ slip systems, which in general may have any character. Following Arsenlis and Parks (2002), we consider dislocation densities of pure edge (e) and screw (s) characters, where $\varrho_{\perp}^{\alpha}$ and $\varrho_{\top}^{\alpha}$ represent the positive and negative edge densities, respectively and $\varrho_{\odot}^{\alpha}$ and $\varrho_{\oplus}^{\alpha}$ represent the positive and negative screw densities, respectively. Associated with the $\alpha^{t h}$ slip system are unit vectors, $\mathbf{n}_{\mathrm{o}}$, normal to the slip plane and $\mathbf{s}_{\mathrm{o}}$ contained within the plane and aligned with the direction of slip. The total dislocation density of each character is given by the respective sums of positive and negative densities, i.e. $\varrho_{\mathrm{e}}^{\alpha}=\varrho_{\perp}^{\alpha}+\varrho_{\mathrm{T}}^{\alpha}$ and $\varrho_{\mathrm{s}}^{\alpha}=\varrho_{\odot}^{\alpha}+\varrho_{\oplus}^{\alpha}$, while the geometrically necessary densities are given by the differences, $\kappa_{\mathrm{e}}^{\alpha}=\varrho_{\perp}^{\alpha}-\varrho_{\odot}^{\alpha}$ and $\kappa_{\mathrm{s}}^{\alpha}=\varrho_{\odot}^{\alpha}-\varrho_{\oplus}^{\alpha}$.

The evolution of plastic deformation is specified using an expression of Orowan's relation, i.e.,

$$
\dot{\mathbf{F}}_{\mathrm{p}} \mathbf{F}_{\mathrm{p}}^{-1}=\widetilde{\mathbf{L}}_{\mathrm{p}}=\sum_{\alpha=1}^{N_{\mathrm{s}}} b^{\alpha}\left(\varrho_{\mathrm{e}}^{\alpha} v_{\mathrm{e}}^{\alpha}+\varrho_{\mathrm{s}}^{\alpha} v_{\mathrm{s}}^{\alpha}\right) \mathbf{s}_{\mathrm{o}}^{\alpha} \otimes \mathbf{n}_{\mathrm{o}}^{\alpha}
$$

where $b^{\alpha}$ is the magnitude of the Burger's vector and $v_{\mathrm{e}}^{\alpha}$ and $v_{\mathrm{s}}^{\alpha}$ are the signed scalar velocities of edge and screw dislocations, respectively, on slip system $\alpha$. The dislocation velocity is expressed in a general form as

$$
v^{\alpha}=\hat{v}\left(\tau^{\alpha}, \varrho^{\beta}, T\right)
$$

where the resolved shear stress on slip system $\alpha$ is $\tau^{\alpha}=J_{\mathrm{e}} \boldsymbol{\sigma}: \mathbf{s}^{\alpha} \otimes \mathbf{n}^{\alpha}, \varrho^{\beta}$ is used to indicate a dependence on the entire field of all dislocation densities including positive, negative, edge, and screw content, in general, and $\beta=\left[1 . . N_{\mathrm{s}}\right]$.

The magnitude of dislocation velocity is defined using the model developed by Austin and McDowell (2011, 2012) and extended to single-crystals by Lloyd et al. (2014) which accounts for thermal activation, viscous drag, and relativistic effects that limit the maximum velocity of dislocations to the shear wave speed of the material. Their model is based on the average velocity at which a dislocation travels between obstacles computed as

$$
v^{\alpha}=\frac{\bar{L}}{t_{\mathrm{w}}+t_{\mathrm{r}}} \operatorname{sign}\left(\tau^{\alpha}-\tau_{\mathrm{b}}^{\alpha}\right)
$$

where $\bar{L}$ is the mean spacing between obstacles, which is simply approximated in this work as $\bar{L}=1 / \sqrt{\varrho_{0}}$ where $\varrho_{0}$ is the initial dislocation density on each slip system. The time a dislocation spends waiting at a barrier, $t_{\mathrm{w}}$, is related to the stress-assisted activation energy required to overcome the barrier and the magnitude of thermal fluctuations. The time the dislocation spends running between obstacles, $t_{\mathrm{r}}$, is related to the drag-limited free-running velocity of a dislocation, i.e., $t_{\mathrm{r}}=\bar{L} / v_{\mathrm{r}}$. Specifically, Austin and McDowell (2011), define

$$
t_{\mathrm{w}}=\frac{1}{\omega}\left(\exp \left[\frac{\Delta G(\tau)}{K T}\right]-1\right)
$$

where $\omega$ is the attempt frequency, $K$ is Boltzmann's constant, and $\Delta G$ is the stress-assisted activation energy. In this case,

$$
\Delta G^{\alpha}(\tau)=\Delta G_{0}\left\langle 1-\left(\frac{\left|\tau^{\alpha}-\tau_{\mathrm{b}}^{\alpha}\right|}{\tau_{\mathrm{cr}}^{\alpha}}\right)^{q_{1}}\right\rangle^{q_{2}}
$$


where $\langle x\rangle=1 / 2(|x|+x), \tau_{\mathrm{b}}^{\alpha}$ is the slip-system back stress due to the geometrically necessary dislocation field and $\tau_{\mathrm{cr}}^{\alpha}$ is the slip resistance on the $\alpha^{t h}$ slip system. The parameters, $q_{1}$ and $q_{2}$ reflect the shape of the energy barrier consistent with the arguments of Kocks et al. (1975). The slip resistance reflects Taylor hardening attributed to the interaction of the total dislocation field, i.e.,

$$
\tau_{\mathrm{cr}}^{\alpha}=\tau_{0}+c_{1} \mu b \sqrt{\sum_{\beta} H_{\alpha \beta}\left(\varrho_{\mathrm{e}}^{\beta}+\varrho_{\mathrm{s}}^{\beta}\right)}
$$

where $\tau_{0}$ is an intrinsic lattice slip resistance, $c_{1}$ is a material constant, and $H_{\alpha \beta}$ is the slip system hardening interaction matrix.

The back stress is computed by

$$
\tau_{\mathrm{b}}^{\alpha}=\widetilde{\mathbf{S}}_{\mathrm{i}}: \mathbf{s}_{0}^{\alpha} \otimes \mathbf{n}_{0}^{\alpha}
$$

where the net internal stress attributed to dislocation configuration, $\widetilde{\mathbf{S}}_{\mathrm{i}}$, is returned as part of the solution to the DDC sub-problem (Section 4).

The run time between obstacles is computed based on a temporal ensemble average of the instantaneous velocities across all dislocations on slip-system $\alpha$. Austin and McDowell (2011) compute the mean freerunning velocity by integrating an assumed spatial velocity profile between obstacles to obtain

$$
v_{\mathrm{r}}^{\alpha}=\frac{\left|\tau^{\alpha}-\tau_{\mathrm{b}}^{\alpha}\right| b}{B^{\star}}
$$

where $B^{\star}$ is a viscous drag coefficient which depends upon the free-running velocity of dislocations, specifically,

$$
B^{\star}=\frac{B_{0}(T)}{1-\left(v_{\mathrm{r}} / c_{s}\right)^{2}}
$$

As $v_{\mathrm{r}}$ approaches the shear wave speed associated with dislocations on a specific slip-system, $c_{\mathrm{s}}$, the effective drag increases without bound. This so-called relativistic effect restricts dislocation velocities to not exceed the current shear wave speed in the material. We use $c_{\mathrm{s}} \approx \sqrt{\bar{\rho} / \mu^{\prime}}$ and, for FCC slip, compute the effective shear modulus on $\{111\}$ planes in $\langle 011\rangle$ directions as $\mu^{\prime}=\left(C_{11}-C_{12}+C_{44}\right) / 3$. Note, the pertinent shear wave speed is anisotropic for single crystals and depends upon temperature and pressure, in general. Austin and McDowell (2011) show the solution of the resulting implicit nonlinear equation is

$$
v_{\mathrm{r}}^{\alpha}=c_{\mathrm{s}}\left(\sqrt{\left(\xi^{\alpha}\right)^{2}+1}-\xi^{\alpha}\right)
$$

where

$$
\xi^{\alpha}=\frac{B_{0}(T) c_{\mathrm{s}}}{2 b\left(\tau^{\alpha}-\tau_{\mathrm{b}}^{\alpha}\right)}
$$

\section{Continuum Dislocation Transport}

The continuum dislocation transport (CDT) sub-problem provides a physical basis for simulating the nonlocal evolution of dislocation fields within the material. A general balance law for conservation of a field, $\varrho$, is

$$
\dot{\varrho}+\nabla_{\mathrm{o}} \cdot \mathbf{f}_{\varrho}=s_{\varrho}
$$

where $\mathbf{f}_{\varrho}$ is the flux of species $\varrho$ and $s_{\varrho}$ is a volumetric source term. Following the arguments of Arsenlis and Parks (1999); Arsenlis et al. (2004); Groma et al. (2003), we employ conservation equations that pertain separately to each component of dislocation density of each dislocation character reflecting polarity and edge/screw character, i.e.,

$$
\dot{\varrho}_{\mathfrak{c}}^{\alpha}+\nabla_{\mathrm{o}} \cdot \mathbf{f}_{\mathfrak{c}}^{\alpha}=s_{\mathfrak{c}}^{\alpha}
$$


which is enforced separately for each slip system, $\alpha$, and dislocation character, i.e., $\forall \mathfrak{c} \in\{\perp, \top, \odot, \oplus\}$. The dislocation fluxes are expressed as

$$
\mathbf{f}_{\mathfrak{c}}=\varrho_{\mathfrak{c}}^{\alpha}\left(\mathbf{v}_{\mathrm{o}}^{\alpha}\right)_{\mathfrak{c}}
$$

We cast the conservation equations within the reference configuration such that the relevant dislocation velocities, $\mathbf{v}_{\mathrm{o}}^{\alpha}$ reflect a plastic pull-back from dislocation velocities computed within the intermediate configuration, i.e., $\left(\mathbf{v}_{\mathrm{o}}^{\alpha}\right)_{\mathfrak{c}}=\mathbf{F}_{\mathrm{p}}^{-1} \mathbf{v}_{\mathfrak{c}}^{\alpha}$, respectively, for each dislocation character. The intermediate configuration dislocation velocities are

$$
\begin{aligned}
& \mathbf{v}_{\perp}^{\alpha}=v_{\mathrm{e}}^{\alpha}\left(\tau^{\alpha}, \varrho^{\beta}, T\right) \mathbf{s}_{\mathrm{o}} \\
& \mathbf{v}_{\top}^{\alpha}=-v_{\mathrm{e}}^{\alpha}\left(\tau^{\alpha}, \varrho^{\beta}, T\right) \mathbf{s}_{\mathrm{o}} \\
& \mathbf{v}_{\odot}^{\alpha}=v_{\mathrm{s}}^{\alpha}\left(\tau^{\alpha}, \varrho^{\beta}, T\right) \mathbf{s}_{\mathrm{o}} \times \mathbf{n}_{\mathrm{o}} \\
& \mathbf{v}_{\oplus}^{\alpha}=-v_{\mathrm{s}}^{\alpha}\left(\tau^{\alpha}, \varrho^{\beta}, T\right) \mathbf{s}_{\mathrm{o}} \times \mathbf{n}_{\mathrm{o}}
\end{aligned}
$$

\section{Constitutive Equations}

In conjunction with the governing equations (29) and dislocation velocity magnitude from Eq. (19), the CDT sub-problem is complete with specification of constitutive equations defining the source term for positive and negative dislocations. In a general form, the dislocation source depends upon stress, temperature, and the dislocation fields including contributions from dislocations of edge and screw character, i.e.,

$$
s^{\alpha}=\hat{s}\left(\sigma, T, \varrho^{\beta}, \kappa^{\beta}\right) \quad \beta=\left[1 . . N_{\mathrm{s}}\right]
$$

The dislocation source terms provide a constitutive interface to introduce models for dislocation interaction (multiplication, annihilation, immobilization, and nucleation). The dependence on these variable fields can be non-local in general; however, here we have used a local constitutive model to account for dislocation multiplication and annihilation. While dislocation nucleation is anticipated under many cases of shock loading, we presently neglect such contributions; this is a challenging problem in its own regard and is beyond the scope of the present work. The framework developed here permits dislocations of edge and screw character and, for general multi-dimensional implementations, the constitutive equations must define source terms that depend upon dislocation density of both edge and screw character. However, for the 1D problems used in this work to demonstrate the utility of the model we simplify the solution by narrowing the problem scope to dislocations of pure edge character. Consequently, in simulations discussed in this paper we have used a simple model for dislocation generation reflecting multiplication and annihilation, i.e.,

$$
s^{\alpha}=\left(M \sqrt{\varrho_{\mathrm{obs}}^{\alpha}} \varrho_{\mathrm{e}}^{\alpha}-A Y_{\mathrm{e}} \varrho_{\perp}^{\alpha} \varrho_{\mathrm{T}}^{\alpha}\right)\left|v_{\mathrm{e}}^{\alpha}\right|
$$

where $M$ and $A$ are the multiplication and annihilation coefficients, respectively, $Y_{\mathrm{e}}$ is a dislocation capture distance and the obstacle density is given by the expression $\varrho_{\mathrm{obs}}^{\alpha}=\sum_{\beta} A_{\alpha \beta} \varrho_{\mathrm{e}}^{\beta}$.

\section{Dislocation Deformation Compatibility}

Internal stress fields are caused by dislocations due to the elastic lattice deformations generated by their presence, and this stress field can either enhance (forward stress) or impede (back stress) slip on particular systems. In a continuum framework where discrete dislocations are not explicitly resolved, such as the one presented in this work, the exact details of the internal stress field cannot be represented since this depends on the nature of the discrete dislocation positions, characters and relative positions. By choosing to represent the dislocation configuration in terms of dislocation densities, these details are not available; however, the long-range internal stress field produced by GNDs can be identified.

Various models exist for identifying the stress field associated with configurations of dislocations. For example, Cottrell (1952) developed an analytical expression for the internal stress field in the vicinity of a 
single edge dislocation. Hirth and Lothe (1969) integrated this stress over a region to identify an internal shear stress per unit edge length acting along a single slip system that will resist or enhance slip caused by the external stress, i.e.,

$$
\tau_{\mathrm{i}}^{\alpha}(z)=\frac{\mu b w}{2 \pi(1-\nu)} \int_{L_{\mathrm{o}}} \frac{\kappa^{\alpha}\left(z^{\prime}\right)}{z^{\prime}-z} d z^{\prime}
$$

where $z$ and $z^{\prime}$ are coordinates along the slip system direction and $w$ is the length along the dislocation line.

Evers et al. (2004a,b) and Yefimov and van der Giessen (2005a,b) use a Taylor series-expansion of this field to obtain a slip-system back stress associated with the gradient of GNDs. Expressing

$$
\kappa^{\alpha}\left(z^{\prime}\right)=\kappa^{\alpha}(z)+\left[z^{\prime}-z\right] \nabla_{\mathrm{s}} \kappa^{\alpha}+\frac{1}{2}\left[z^{\prime}-z\right]^{2} \nabla_{\mathrm{s}}^{2} \kappa^{\alpha}+\ldots
$$

where the ellipsis represents higher order terms and $\nabla_{\mathrm{s}}$ is the directional derivative along the slip direction. Truncating Eq. (38) to first two terms, substituting into Eq. (37), and assigning a characteristic length $w=L_{\mathrm{o}}$ yields

$$
\tau_{\mathrm{i}}^{\alpha}(z)=\frac{\mu b}{2 \pi(1-\nu)} L_{\mathrm{o}}^{2} \nabla_{\mathrm{s}} \kappa^{\alpha}(z)
$$

thus providing a weakly nonlocal internal stress that depends upon the GND field only via its gradient. Eqs. (37) and (39) do not reflect the energetic interaction of dislocations on differing slip systems. A generalization of the GND gradient-based back stress was developed by Yefimov and van der Giessen (2005a) and Bayley et al. (2006). Essentially, the contribution to the total internal stress is separated by slip system, for example,

$$
\widetilde{\mathbf{S}}_{\mathrm{i}}=\sum_{\alpha=1}^{N_{\mathrm{s}}} \tau_{\mathrm{i}}^{\alpha} \mathbf{M}_{\mathrm{o}}^{\alpha}
$$

where the contribution to internal stress from the $\alpha^{\text {th }}$ slip system is based on Eq. (39). Then the effective back stress computed by projection (cf. Eq. 23) reflects the energetic interaction between the dislocations on varying slip systems. Several alternatives for the basis tensor, $\mathbf{M}_{\mathrm{o}}^{\alpha}$, were investigated by Yefimov and van der Giessen (2005a), for example,

$$
\mathbf{M}_{\mathrm{o}}^{\alpha}=\frac{1}{2}\left(\mathbf{s}_{\mathrm{o}}^{\alpha} \otimes \mathbf{n}_{\mathrm{o}}^{\alpha}+\mathbf{n}_{\mathrm{o}}^{\alpha} \otimes \mathbf{s}_{\mathrm{o}}^{\alpha}\right)
$$

Bayley et al. (2006) expand this idea to normal stress contributions from dislocations in the lattice and provide sets of base tensors to reflect contributions from both edge and screw dislocations.

While previous work of Evers et al. (2004a,b); Yefimov and van der Giessen (2005a,b); Bayley et al. (2006), for example, have developed strategies for including the back stress computed from the dislocation density fields, they do not attempt to include the corresponding elastic lattice strain fields reflected by $\mathbf{F}_{\mathrm{i}}$ of Eq. (4). In an alternate related approach, Gerken and Dawson (2008) use the Volterra solution for stress in the vicinity of a dislocation and isotropic elasticity to relate an internal strain field $\boldsymbol{\epsilon}_{\mathrm{i}}$ to slip gradients associated with GNDs. The total stress contributing to linear momentum conservation is the linear superposition of both the internal stress and the elastic stress, associated with the internal strain and small elastic strain, respectively. Their approach reflects a consistency between lattice strain and stress that is missing in prior works focused on relating internal back stress to GND content.

In this work, we adopt an ad-hoc extension of the back-stress theories of Yefimov and van der Giessen (2005a) and Bayley et al. (2006) that includes a description of strain in a conceptually similar manner to Gerken and Dawson (2008). Specifically, we use Eqs. (39) and (40) with (41) to compute the internal stress. The internal strain associated with the dislocation configuration is then computed from the elastic compliance, i.e.,

$$
\boldsymbol{\epsilon}_{\mathrm{i}}=\mathbb{C}^{-1}: \widetilde{\mathbf{S}}_{\mathrm{i}}
$$

and, making an assumption of small internal elastic strains, is related to a corresponding deformation map via

$$
\mathbf{F}_{\mathrm{i}}=\mathbf{I}+\boldsymbol{\epsilon}_{\mathrm{i}}
$$




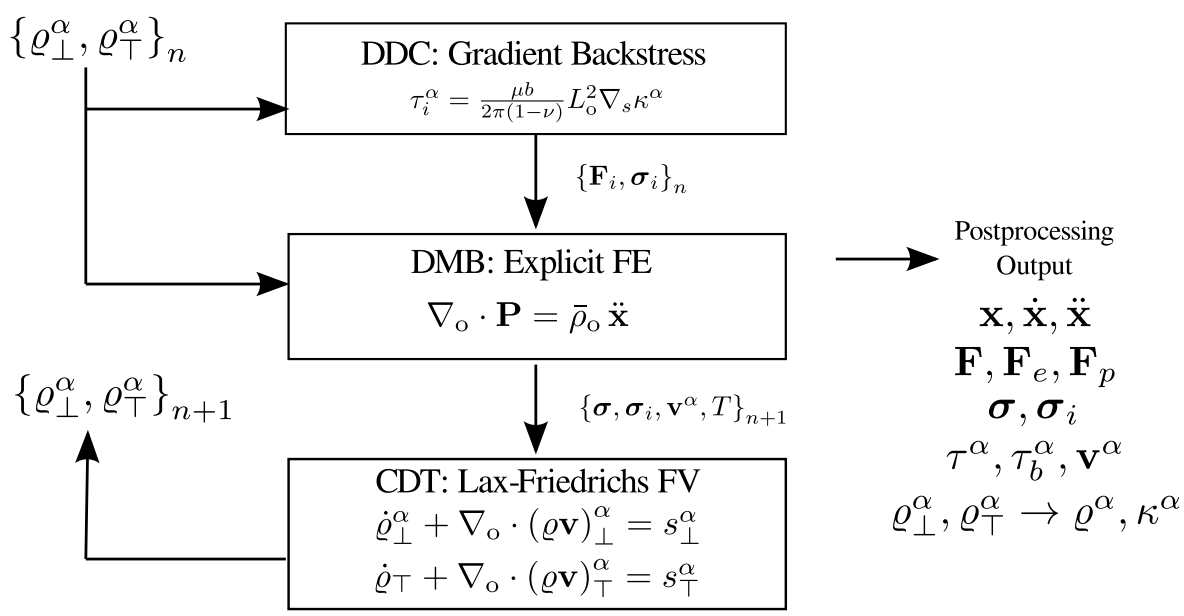

Figure 2: Schematic diagram of explicit single-pass coupling scheme used in simulations discussed here. Dislocation densities from the previous time step are used to solve the DDC and DMB sub-problems. Resulting stress and dislocation velocities fields are subsequently used to update CDT sub-problem.

\section{Numerical Simulations}

Mayeur et al. (2015) present details of the numerical implementation used within simulations discussed in this work. Accordingly, only a brief description of the numerical scheme is provided here. The DMB sub-problem is solved using a total Lagrange finite element spatial discretization. Integration in space is performed using single point Gauss quadrature and linear shape functions to interpolate deformation fields, while explicit time integration is used to advance the deformation fields in time. An artificial bulk viscosity identical to that of Luscher et al. (2013) is added to the quasi-conservative stress field. Linear and quadratic bulk viscosity coefficients are 0.2 and 1.2 respectively. Crystal plasticity equations are updated using fourth order Runge-Kutta explicit integration in time with sub-incrementation used to ensure that the estimated percent error in slip rate remains below a tolerance of $10^{-3}$.

Mourad et al. (2015) implement and compare the performance of several numerical schemes for the CDT sub-problem. Here we use a local Lax-Friedrichs finite-volume scheme which selectively adds diffusivity to solve Eqs. (29). The DDC sub-problem is solved by linearly interpolating GND densities from the finite volume cell centers to cell edges and then using the corresponding finite-element shape functions from the DMB sub-problem to compute GND gradients (cf. Eq. 39) at finite element integration points.

Figure 2 illustrates the staggered explicit scheme used to solve the coupled CDT, DMB, and DDC subproblems. The DDC sub-problem is solved using the dislocation fields from time $t=t_{n}$, thus providing both the internal deformation and internal stress fields needed for the constitutive update of the DMB subproblem, cf. Eqs. (4) and (23). The DMB sub-problem is advanced in time via a central-difference explicit scheme using the dislocation density field from time $t_{n}$, and the internal stress and associated internal deformation fields computed by the DDC sub-problem at time $t_{n+1}$. The dislocation velocities at time $t_{n+1}$ are computed as part of the DMB sub-problem and used to advance the CDT sub-problem from time $t_{n}$ to $t_{n+1}$. This explicit coupling scheme performs satisfactorily because of the extremely small time steps used for the explicit Lagrange solution of the DMB sub-problem. Mourad et al. (2015) have investigated cases where alternate strategies may be advantageous. For example, fully implicit or higher-order explicit coupling schemes may be advantageous when the dislocation fields are evolving rapidly and the changes in back stress are large in comparison to the magnitude of total stresses. The explicit coupling strategy proved sufficient for the simulations discussed in this paper.

Simulations of a classic flyer plate impact experiment are presented in an effort to highlight the intended applicability of the model. The simulation cases are primarily illustrative and, as such, will not be compared directly to any specific experiment. Figure 3 illustrates the basic problem modeled in the first of these simulations. A flyer plate is initially traveling at a velocity $u_{0}$ until it impacts a target specimen plate comprising 
single-crystal copper whose [100] plane normal is aligned with the direction of shock propagation. We model this as a one-dimensional plane wave and neglect reflections off of the traction-free outer circumference based on the assertion that the specimen diameter is large compared to thickness. Assuming a symmetric impact such that the flyer and target plates comprise the same material, material points on the surface of the target specimen are accelerated to a velocity $u_{\mathrm{i}}=u_{0} / 2$. A depiction of the $1 \mathrm{D}$ solution grid is provided in the lower left of Figure 3. The DMB boundary conditions are

$$
\begin{cases}\dot{x}_{1}\left(X_{1}=0, t\right)=u_{\mathrm{i}}, & \text { for } t<t_{\mathrm{f}} \\ \sigma_{11}=0, & \text { otherwise }\end{cases}
$$

where the boundary velocity condition is replaced by traction-free condition at $t=t_{\mathrm{f}}$ in order to simulate a release wave. The CDT sub-problem is solved employing zero flux boundary conditions, i.e.,

$$
v_{1}(0, t)=v_{1}(L, t)=0
$$

The zero flux boundary condition on free surfaces of the target plate does not reflect a common physical scenario, but is a useful bounding case for evaluating the theory. One could envision specialized physical cases that would be reasonably approximated by this condition (e.g. passivated layers on free surface of target plate). A more general case that allows dislocations to freely exit the domain, but prohibits their entry (other than through source terms) is reflected by

$$
\mathbf{v}_{\mathrm{o}}^{\alpha}(0, t) \cdot \mathbf{N}_{\mathrm{o}}(0) \geq 0 \quad \text { and } \quad \mathbf{v}_{\mathrm{o}}^{\alpha}(L, t) \cdot \mathbf{N}_{\mathrm{o}}(L) \geq 0
$$

where $\mathbf{N}_{\mathrm{o}}$ is the boundary surface normal in the reference configuration. These conditions are not included in simulations discussed here, but will be considered in future work. The model parameters used for the simulations in this section are provided in Table 2. References are provided in Table 2 where the corresponding parameter was taken directly from the literature. For other parameters, nominal values were selected from reasonable corresponding ranges without any deliberate attempts to fit experimental data. Consequently, these model parameters are only notionally representative of copper single crystal behavior.

A comparison of dislocation velocity and the viscous drag coefficient computed from Eqs. (19) - (25) for the parameters used in this work (cf. Table 2) with results for molecular dynamics simulations from Wang and Beyerlein (2008) is provided in Figure 4. Wang and Beyerlein (2008) performed molecular dynamic simulations to understand the effects of dislocation motion on stacking fault widths for screw dislocations in $\mathrm{Cu}$. Although this work only includes edge dislocations, the comparison in Fig. 4 demonstrates that the listed parameters are reasonable for representing the response of single crystal copper.

The right side of Figure 3 provides a schematic illustrating the nominal transient response immediately subsequent to impact. At an early time, $t=t_{1}$ the compression wave has traveled some distance through the thickness of the specimen. The imposed uniaxial deformation is immediately accommodated by elastic deformation generating large pressure, as well as deviatoric stress that drives dislocation motion. A notional configuration of edge dislocations along a slip system (projected onto the $X_{1}$ axis) at the corresponding time is depicted in Figure 3. Positive and negative dislocations move in opposite directions under the applied stress as illustrated in the figure. Under the condition of zero dislocation flux through the impact interface, dislocations pile-up and create a back stress that quickly shuts off plasticity at the boundary. At some later time, $t=t_{2}>t_{1}$, the compression wave has progressed further into the specimen and the GND density profile consists of a single well (or hump depending on slip-system orientation) profile traveling with the shock wave that has separated from the GND pile-up remaining at the zero-flux interface.

The athermal limit is an effective stress of approximately $100 \mathrm{MPa}$. Figure 5 provides simulation results consistent with the schematic response depicted in Figure 3. Spatial profiles of uniaxial compressive stress (top), $\sigma_{11}$, and GND density on one slip system (bottom) are plotted across the thickness of the specimen at three different times to compare the relationship between the compression and GND wave profiles. The distribution of GNDs leads to a back-stress distribution comprising a dominant peak at the boundary associated with the pile-up that is resisting slip, in addition to the expected well (assisting slip) at the front of the plastic wave and resisting slip at the tail of the plastic wave. The back-stress profile on the corresponding 
Table 2: Values of model parameters used for the simulations discussed in this work.

\begin{tabular}{|c|c|c|c|}
\hline Parameter & Value & Unit & Reference \\
\hline $\bar{\rho}_{\mathrm{o}}$ & $8.96 \times 10^{3}$ & $\mathrm{~kg} / \mathrm{m}^{3}$ & Greeff et al. (2006) \\
\hline$C_{11}$ & 169.0 & $\mathrm{GPa}$ & Luscher et al. (2013) \\
\hline$C_{12}$ & 122.0 & $\mathrm{GPa}$ & Luscher et al. (2013) \\
\hline$C_{44}$ & 75.3 & $\mathrm{GPa}$ & Luscher et al. (2013) \\
\hline $\mathrm{v}_{0}$ & 0.1119 & $\mathrm{cc} / \mathrm{g}$ & Greeff et al. (2006) \\
\hline $\mathrm{v}^{*}$ & 0.1101 & $\mathrm{cc} / \mathrm{g}$ & Greeff et al. (2006) \\
\hline$B^{*}$ & 143.7 & $\mathrm{GPa}$ & Greeff et al. (2006) \\
\hline$B_{1}$ & 5.25 & - & Greeff et al. (2006) \\
\hline$a$ & 1.259 & - & Greeff et al. (2006) \\
\hline$b$ & 0.0747 & - & Greeff et al. (2006) \\
\hline$\gamma^{\infty}$ & 0.6667 & - & Greeff et al. (2006) \\
\hline$T_{D 0}$ & 321.7 & $\mathrm{~K}$ & Greeff et al. (2006) \\
\hline$\Gamma_{0}$ & 11.13 & $\mathrm{~mJ} / \mathrm{kg}-\mathrm{K}$ & Greeff et al. (2006) \\
\hline$\kappa_{\mathrm{S}}$ & 0.717 & - & Greeff et al. (2006) \\
\hline$c_{1}$ & 0.5 & - & - \\
\hline$\varrho_{0}$ & $10^{9}$ & $\left(\mathrm{~mm} / \mathrm{mm}^{3}\right)$ & - \\
\hline$|\mathbf{b}|$ & $3 \times 10^{-7}$ & $\mathrm{~mm}$ & - \\
\hline$B_{0}$ & $3 \times 10^{-11}$ & MPa-s & - \\
\hline$g_{0}$ & 0.87 & - & Austin and McDowell (2012) \\
\hline$\omega_{0}$ & $8 \times 10^{11}$ & $1 / \mathrm{s}$ & - \\
\hline$p$ & 0.33 & - & Bronkhorst et al. (2007) \\
\hline$q$ & 1.66 & - & Bronkhorst et al. (2007) \\
\hline$\tau_{0}$ & 10.0 & $\mathrm{MPa}$ & Bronkhorst et al. (2007) \\
\hline$K_{\mathrm{s}}$ & 30 & - & - \\
\hline$A_{\mathrm{s}}$ & 2 & - & - \\
\hline$Y_{\mathrm{e}}$ & $6|\mathbf{b}|$ & - & - \\
\hline$A_{\text {int }}$ & 0.1 & - & - \\
\hline
\end{tabular}

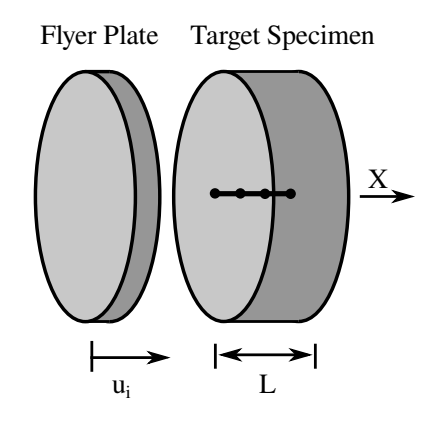

Diagram of Response in Target Plate
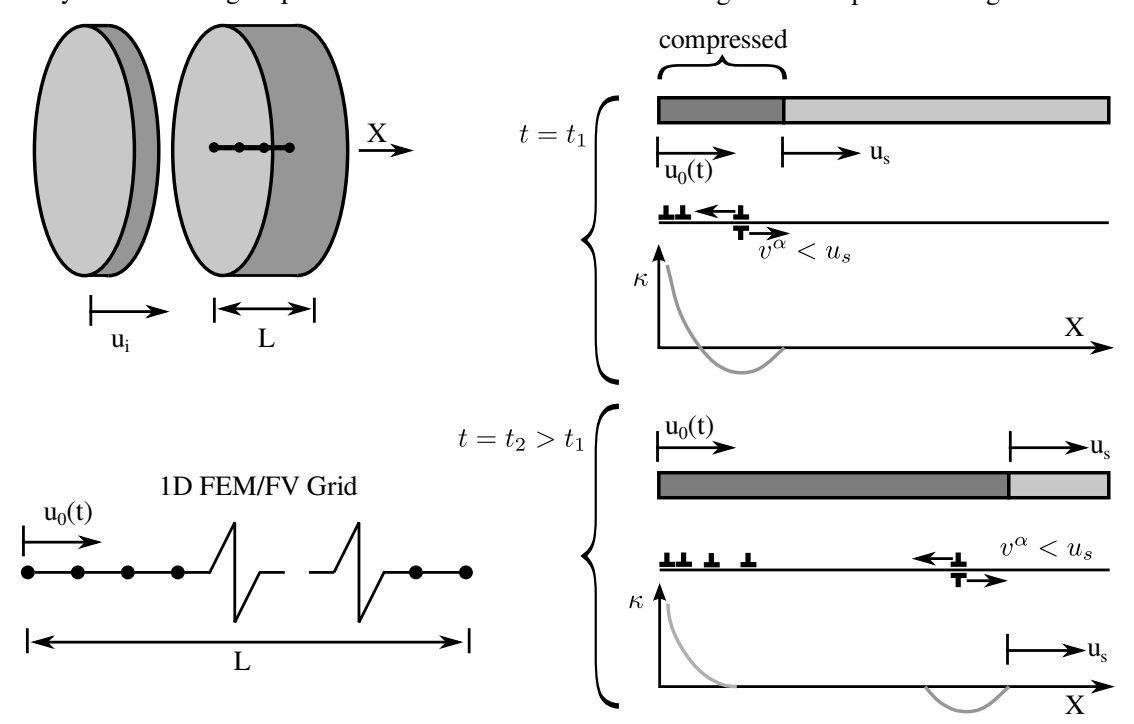

Figure 3: Illustration of plate impact problem, numerical grid, and cartoon of dislocation and shock wave motion. Note, the flyer plate is not explicitly resolved in the calculations and is not depicted in the mesh nor diagram of response. 


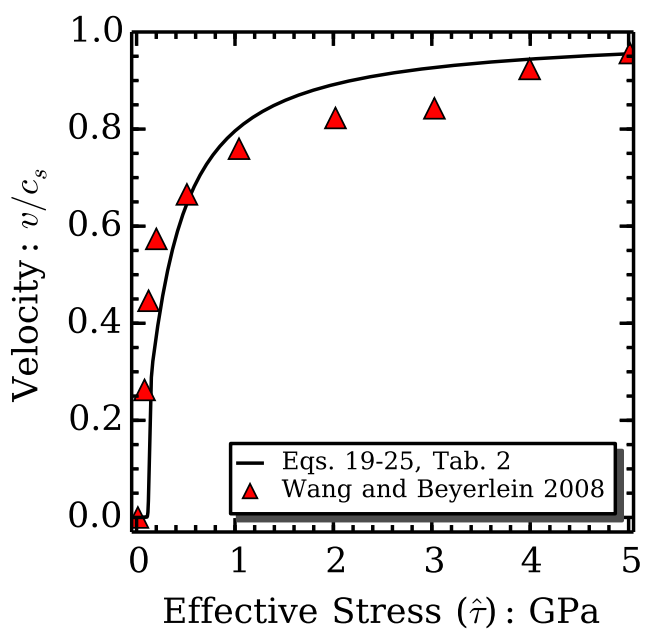

(a) dislocation velocity

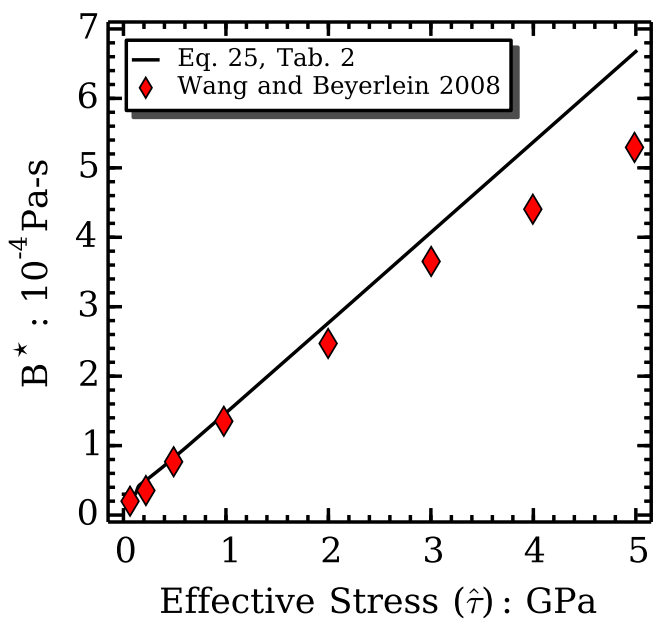

(b) dynamic drag coefficient

Figure 4: Comparison of (a) dislocation velocity and (b) drag coefficient for model parameters used in this work (cf. Table 2) and results of molecular dynamics simulations of screw dislocations in $\mathrm{Cu}$ from Wang and Beyerlein (2008).

slip system is plotted in blue at time $t=0.1 \mu \mathrm{s}$ on the left side of Figure 6 . The associated distribution of projected shear stress is plotted in black and the net effective shear stress in red. Clearly, the net effective stress is limited within the plastic wave to a value just above the athermal limit observed in Figure 4. Consequently, the total externally applied shear stress, $\tau^{\alpha}$ reflects the peaks from the back-stress. The subtle separation of the external stress wave into an elastic precursor and plastic wave is evident by the slight jog in the rising edge of the external shear stress profile. The slip velocity profile on this slip system (all systems behave identically after consideration of sign) is depicted on the right side of Figure 6 for times coincident with those in Figure 5.

Next we simulate the progression of a compression wave, subsequent release waves, and the consequential tensile pulse (such as might lead to spallation) across a target plate impact specimen. Embedded at the mid-point $\left(X_{1}=L / 2\right)$ of the simulation domain, $0 \leq X_{1} \leq L$, is a zero-flux interface which represents a hypothetical grain boundary that is impermeable by dislocations. A diagram of this case is included in Figure 7. The compression wave is initiated using the same DMB boundary conditions as for the prior case where the traction free boundary condition is specified at $t=t_{\mathrm{f}}=0.24 \mu \mathrm{s}$. Again in this problem, zero-flux boundary conditions are employed at the left and right boundaries of the CDT sub-problem. In addition, a zero-flux interface condition is imposed via $\mathbf{v}_{\mathrm{o}}^{\alpha}\left(X_{1}=L / 2, t\right) \cdot \mathbf{N}_{\mathbf{l}}=\mathbf{0}$ where $\mathbf{N}_{1}$ is a unit vector normal to the interface at $X_{1}=L / 2$.

The simultaneous progression of the uniaxial compressive stress wave and GND density profile is plotted for $t<t_{\mathrm{f}}$ in Figure 8. The GND profile exhibits the expected well following the plastic wave prior to the compression wave reaching the interface. After the shock wave transits the zero-flux interface, GNDs are piled up at the boundary causing a back stress resisting plasticity at the interface.

Figure 9 includes plots of the distribution of uniaxial component of deviatoric stress at various times during initial shock passage (left) and during the formation of tensile pulse (right). Solid lines are used to indicate the external stress, while dotted lines represent the internal stress from GND distribution. During the initial shock loading, the deviatoric stress separates into an elastic precursor and trailing plastic wave. This behavior is evident from the evolution of the leading edge of the stress profile. The large back stress built up at the zero-flux interface gives rise to a large external stress at the same location, again, because the net effective stress will remain near the athermal limit after passage of the plastic wave.

The interception of release waves reflected from free surfaces, an effect simulated on the impact surface by removal of the velocity boundary condition at $t=t_{\mathrm{f}}$, leads to the development of a tensile region containing the zero-flux interface. The time evolution of the spatial distribution of the uniaxial deviatoric stresses are 

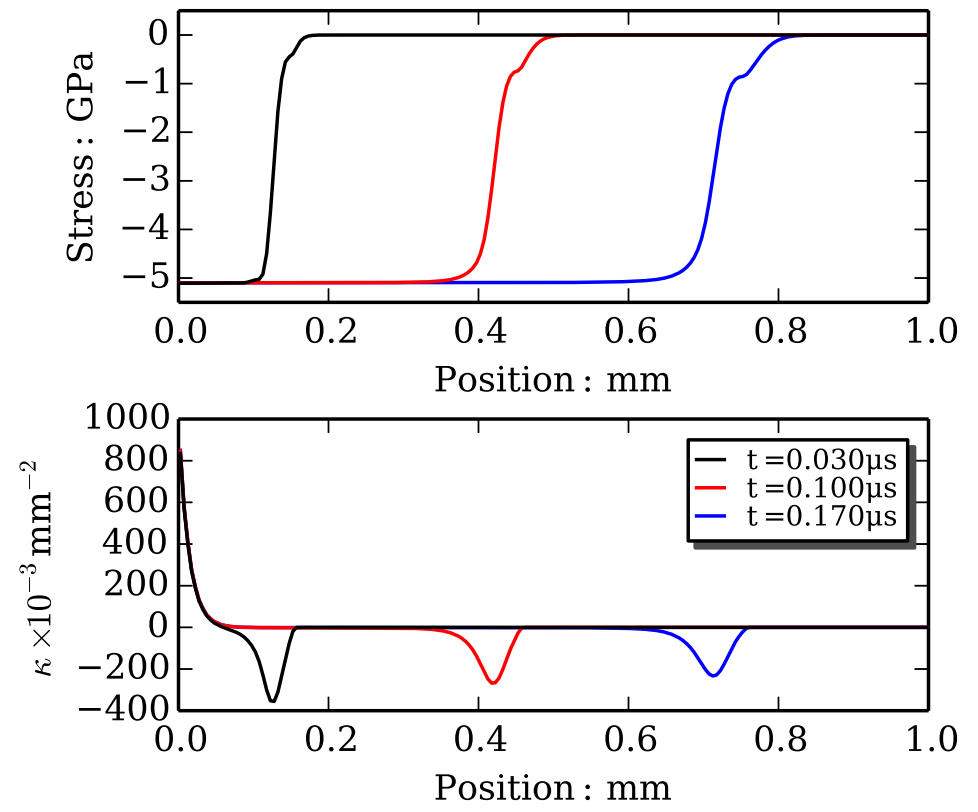

Figure 5: Simulation results illustrating the progression of (top) axial stress and (bottom) GND density wave profiles after impact. The breakout of an elastic precursor is evident in the uniaxial stress profile. A dislocation pile up forms at the left boundary and remains as the GND profile across the wave front transits through the target plate in order to accommodate the gradient in plastic deformation .
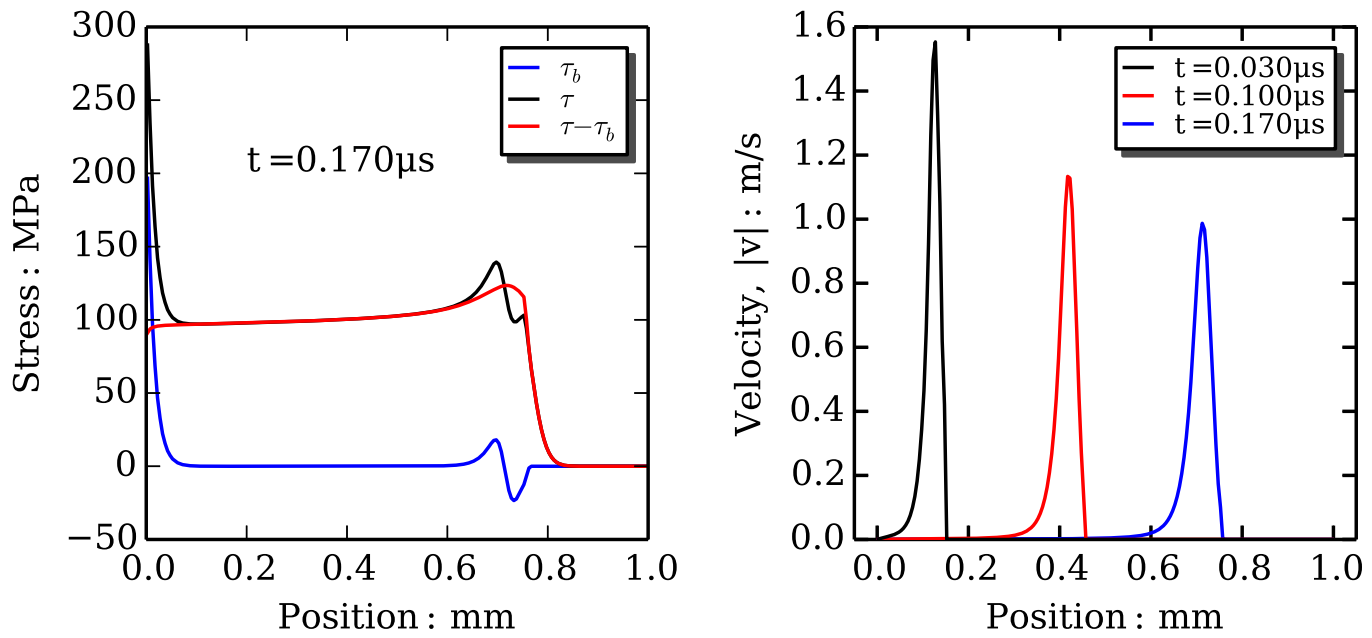

Figure 6: Simulation results illustrating (left) total stress, back stress, and effective driving stress at $t=0.17 \mu s$ and (right) the associated development of dislocation velocity wave. The dislocation pile up at the left boundary has resulted in a pronounced backstress at that location. The effective stress rises sharply at the front of the plastic wave and relaxes in its wake. Consequently, the dislocation velocity profile across the plastic wave has a sharp front and more diffuse trailing edge. 


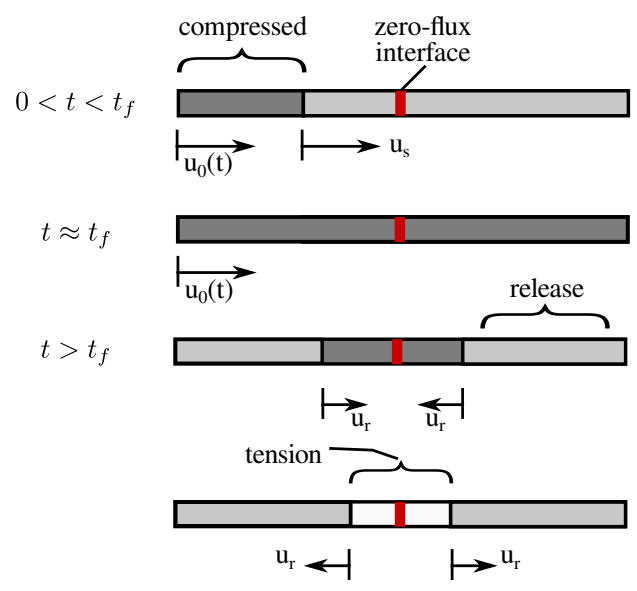

Figure 7: Illustration of model interface problem. Only the target plate is depicted. At early times $t<t_{\mathrm{f}}$ a compression wave transits through the thickness. Impact velocity boundary conditions are removed at $t \approx t_{\mathrm{f}}$ so that the subsequent release waves from both faces impinge and develop a tensile pulse nearly centered at the zero dislocation-flux interface (red).

plotted on the right side of Figure 9. At $t=0.3 \mu$ s the uniaxial deviatoric stress is tensile throughout much of the simulation domain remote from the interface; however, both the internal and external deviatoric stresses remain compressive at the interface where the significant internal stresses serve to resist slip. At $t=0.35 \mu \mathrm{s}$ the response at the interface has changed significantly because of the evolving external stress field. At this time, the external deviatoric stress has become tensile and the internal stress, largely unchanged from $t=0.30 \mu \mathrm{s}$ is acting to favor slip in the reverse direction from that during the initial compressive loading. This reversal in stress drives dislocations in the opposite direction eventually leading to GND pile up of opposite sense and a change in sign of internal stress at the interface. Profiles of total accumulated plastic slip summed over all slip systems are shown in Figure 10 at various times throughout the release wave and tensile interaction process. The asymmetry in these profiles is caused in part by the non-symmetric timing of the interception of release waves and in part by the diffusion (both artificial and material) of the incident shock wave.

Finally, we demonstrate the potential utility of the modeling approach to problems where the DMB sub-problem domain does not coincide with the CDT and DDC sub-problem domain. For example, we envision application to polycrystal problems where the DMB sub-problem is necessarily solved over the entire polycrystal domain, but the CDT and DDC sub-problems need only be solved in the vicinity of regions that would affect dislocation motion, e.g., adjacent to grain boundaries. A representative problem highlighting this potential application is depicted in Figure 11. The propagation of a shock wave through the single crystal is modeled by solving the DMB sub-problem over the domain $-L / 2<X_{1}<3 L / 2$ and the CDT and DDC sub-problems over the domain $0<X_{1}<L$. The DMB boundary conditions are $\dot{x}_{1}\left(X_{1}=-L / 2, t\right)=u_{\mathrm{i}} / 2$ and CDT boundary conditions are zero flux in and out of the CDT sub-domain, i.e., $\left.\left(\mathbf{v}_{\mathrm{o}}^{\alpha} \cdot \mathbf{N}_{\mathrm{o}}\right)\right|_{X_{1}=0}=\left.\left(\mathbf{v}_{\mathrm{o}}^{\alpha} \cdot \mathbf{N}_{\mathrm{o}}\right)\right|_{X_{1}=L}=0$

Figure 12 illustrates the progression of the uniaxial stress and GND profile on a representative slip system during passage of a shock wave through the CDT sub-domain. At time $t=0.1 \mu \mathrm{s}$ the wave has not entered into the CDT domain and, thus, there is zero GND density. At $t=0.2 \mu$ s the wave is transiting the CDT domain leading to dislocation pile-up at the left domain boundary along with the previously observed traveling GND profile associated with the plastic wave. At time $t=0.4 \mu$ s the wave has exited the CDT domain and the GND distribution reflects an essentially anti-symmetric pile-up condition at both interfaces. Spatial profiles of the projected shear stress, back stress, and relative effective stress corresponding with this GND distribution and stress state are plotted in Figure 13. The back-stress distribution is such that it has resisted slip at the CDT domain boundary during passage of the shock wave. The effective driving stress has relaxed through viscoplastic slip to just below the athermal limit of the dislocation velocity model (cf. Fig. 4). Consequently, the profile of projected (external) shear stress peaks at the zero-flux boundaries. 

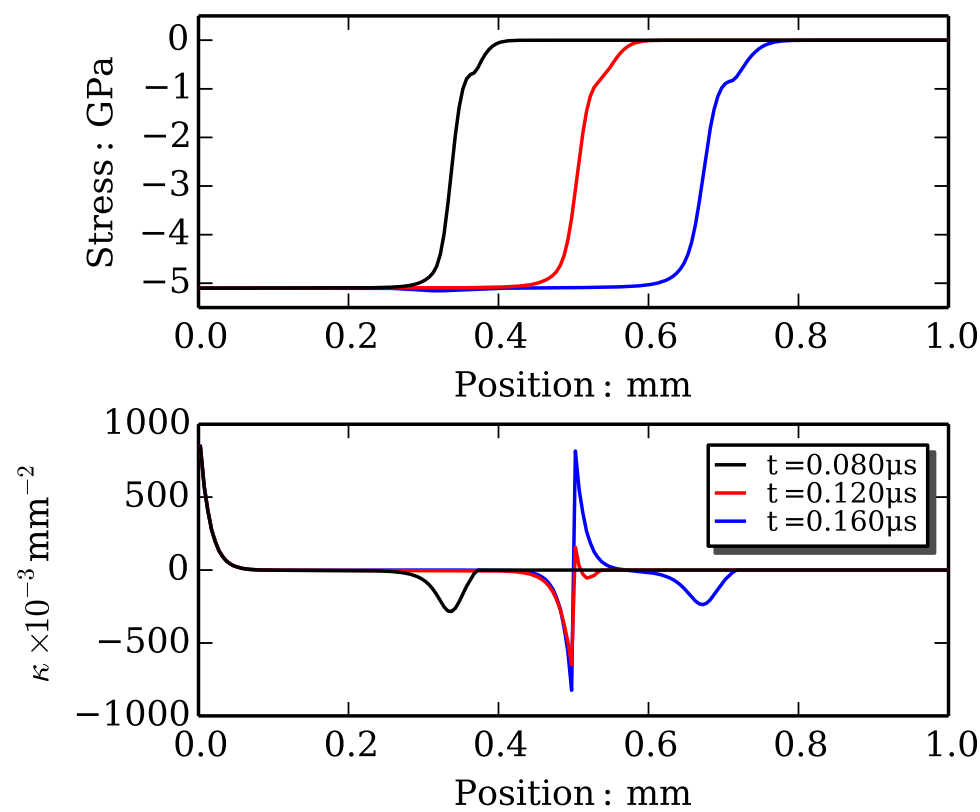

Figure 8: Plot of uniaxial stress profile (top) and simultaneous GND distribution at various times during the initial passage of compressive wave through target plate with embedded zero dislocation-flux interface. Dislocations pile up at embedded interface, in addition to left and right boundaries, restricting plastic flow at those locations.

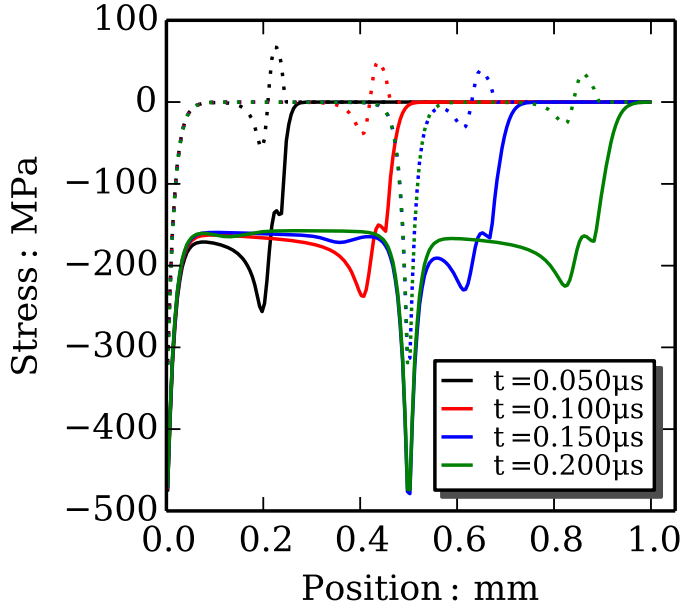

(a) compressive shock loading

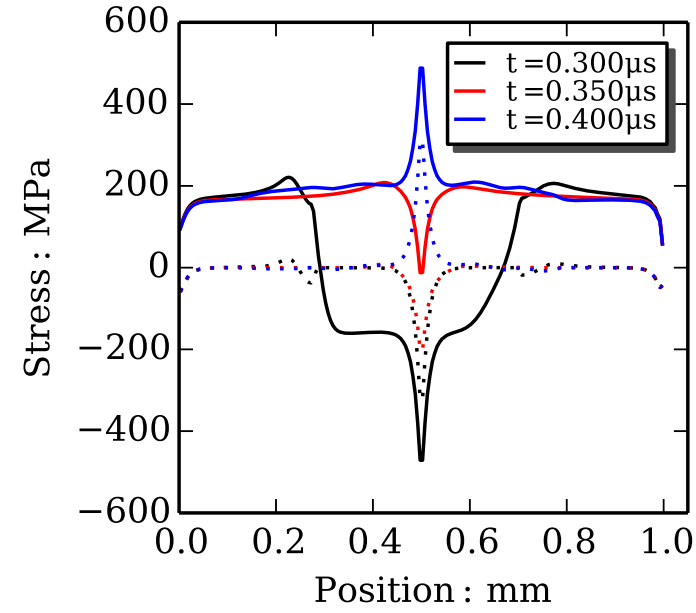

(b) intersection of release waves and tensile pulse.

Figure 9: Plots of uniaxial deviatoric stress profile at various times. Solid lines indicate total external stress and dashed lines represent the internal stress associated with dislocation configuration. After the compression wave has transited the embedded interface, a subtle recompression wave that has reflected off the embedded interface can be observed (blue and green curves on left). 


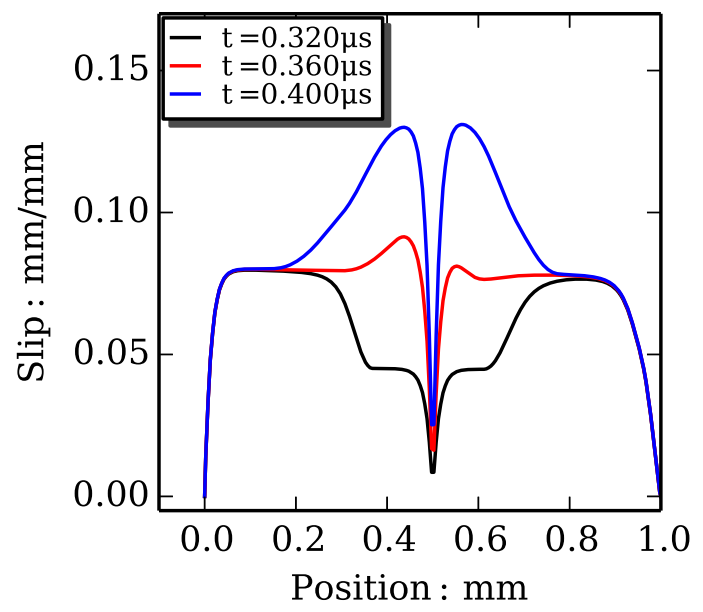

Figure 10: Plot of spatial distribution of total accumulated slip though the specimen thickness at various times. The tensile pulse accentuates accumulating slip in late times except in the near vicinity of the zero dislocation-flux interface which prohibits slip across the interface.

\section{D dual-domain FV / FEM example}

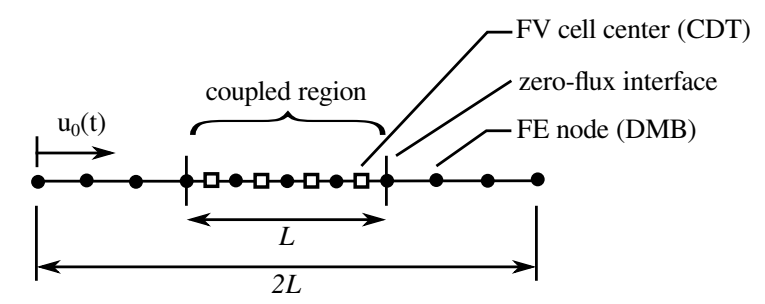

Figure 11: Illustration of mixed-domain 1D example problem where the CDT sub-problem is solved only over a portion of the entire DMB domain. This example case alludes to potential applications demanding fully-coupled physics within the vicinity of material interfaces, while permitting computationally expedient solutions of DMB sub-problem at locations remote from such interfaces.

The slight upward slope of the external and effective stresses throughout the central region of the CDT sub-problem are associated with the time scale of continued stress relaxation from viscoplastic slip.

\section{Concluding Remarks}

In this work we have presented a novel approach for modeling the role of dislocations in continuum dynamic simulations of shock loading of materials. The model framework couples a theory for continuum dislocation transport (CDT) with traditional Lagrange computational schemes for solving the conservation of linear momentum, referred to here as a deformation momentum balance (DMB) sub-problem. The nonlocal interaction of dislocations arises through the dislocation-deformation compatibility (DDC) sub-problem which represents a solution for the distribution of interaction forces and the associated kinematic consequence of the elastic lattice strains of a particular dislocation configuration. In the specific model presented here, we employ a weakly nonlocal model for internal stress based on the gradient of GND density on each particular slip-system. In order to highlight the utility and applicability of the framework, results from simulations of three notional model problems are presented. While the selected cases are idealized for illustrative purposes, the model parameters used here are generally consistent with the shock loading of single crystal copper along a [100] surface normal. 

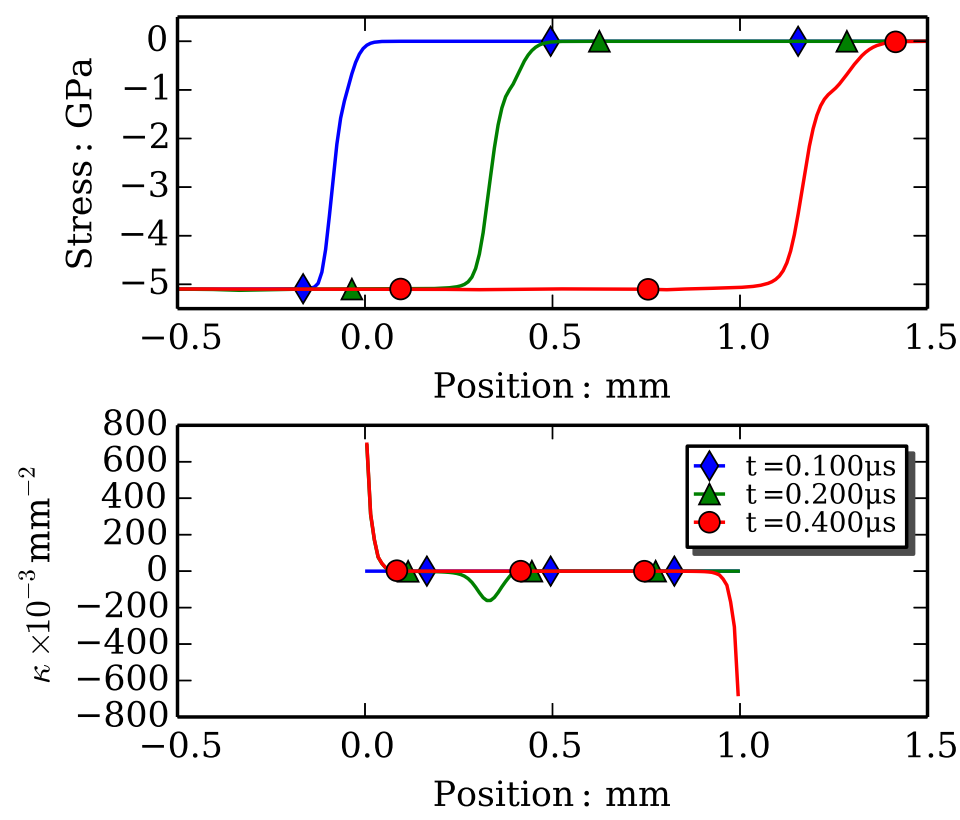

Figure 12: Plots illustrating the progression of the uniaxial stress (top), $\sigma_{11}$, and representative GND, $\kappa$, profile during passage of a shock wave through the CDT sub-domain.

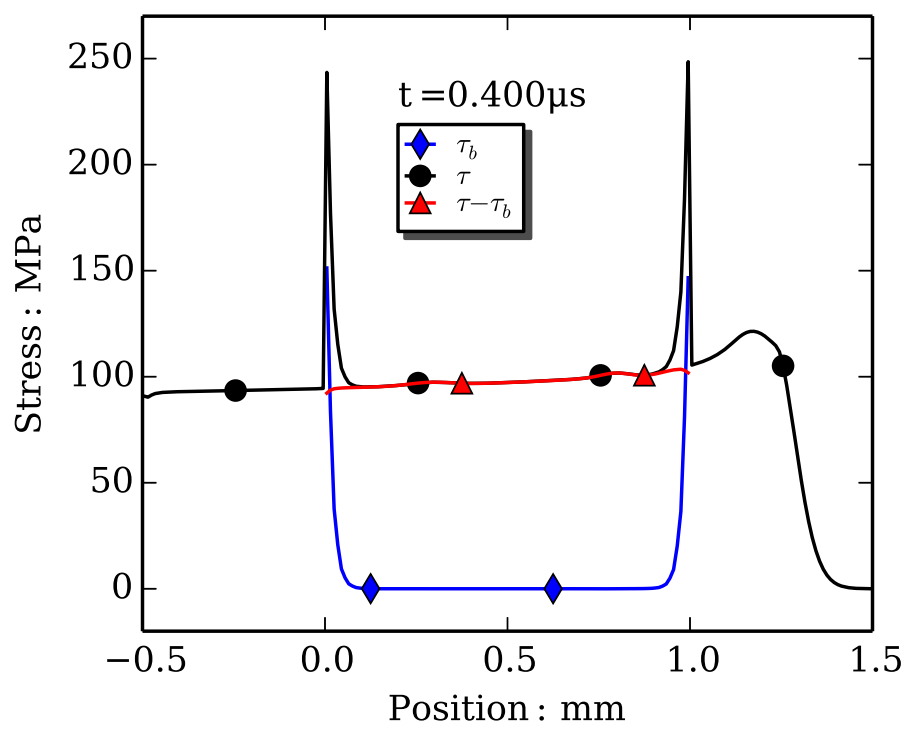

Figure 13: Plot of the projected shear stress (black), back stress (blue), and relative effective stress (red) on a representative slip system after passage of the shock wave through CDT sub-domain. 
We believe the theoretical framework and modeling approach presented here will prove instrumental for simulating the effects of interfaces within single- and poly-crystals subjected to shock and impact loading. We have demonstrated the approach for plane wave impact loading of single crystals. The extension to polycrystals is conceptually straightforward and the approach enables direct introduction of interfacial constitutive relations or boundary conditions via the dislocation velocity and source models that can depend upon interface characteristics which, in general, may evolve throughout the solution. Future extensions of the approach will include generalization to multiple dimensions and implementation of an enhanced kinematic framework for an improved solution to the DDC sub-problem.

\section{Acknowledgments}

Insightful comments made by the reviewers were helpful in improving the communication of this work. The authors gratefully acknowledge the support of the U.S. Department of Energy through the LANL Laboratory Directed Research Development Program (LDRD) and, in particular, funding for the exploratory research project (LDRD-ER-140645) on modeling Materials for the Future.

\section{References}

Acharya, A., Apr. 2001. A model of crystal plasticity based on the theory of continuously distributed dislocations. Journal of the Mechanics and Physics of Solids 49 (4), 761-784.

Acharya, A., Jun. 2003. Driving forces and boundary conditions in continuum dislocation mechanics. Proceedings of the Royal Society A-mathematical Physical and Engineering Sciences 459 (2034), 1343-1363.

Acharya, A., Mar. 2007. Jump condition for gnd evolution as a constraint on slip transmission at grain boundaries. Philosophical Magazine 87 (8-9), 1349-1359.

Acharya, A., Roy, A., Aug. 2006. Size effects and idealized dislocation microstructure at small scales: Predictions of a phenomenological model of mesoscopic field dislocation mechanics: Part i. Journal of the Mechanics and Physics of Solids 54 (8), 1687-1710.

Aghababaei, R., Joshi, S. P., Reddy, J., 2011. Nonlocal continuum crystal plasticity with internal residual stresses. Journal of the Mechanics and Physics of Solids 59 (3), 713-731.

Alankar, A., Eisenlohr, P., Raabe, D., 2011. A dislocation density-based crystal plasticity constitutive model for prismatic slip in $\alpha$-titanium. Acta Materialia 59 (18), 7003-7009.

Arsenlis, A., Parks, D. M., Mar. 1999. Crystallographic aspects of geometrically-necessary and statisticallystored dislocation density. Acta Materialia 47 (5), 1597-1611.

Arsenlis, A., Parks, D. M., Sep. 2002. Modeling the evolution of crystallographic dislocation density in crystal plasticity. Journal of the Mechanics and Physics of Solids 50 (9), PII S0022-5096(01)00134-X.

Arsenlis, A., Parks, D. M., Becker, R., Bulatov, V. V., Jun. 2004. On the evolution of crystallographic dislocation density in non-homogeneously deforming crystals. Journal of the Mechanics and Physics of Solids 52 (6), 1213-1246.

Asaro, R. J., 1983. Crystal plasticity. Journal of applied mechanics 50 (4b), 921-934.

Ashby, M. F., 1970. Deformation of plastically non-homogeneous materials. Philosophical Magazine 21 (170), 399-\&.

Aslan, O., Cordero, N., Gaubert, A., Forest, S., 2011. Micromorphic approach to single crystal plasticity and damage. International Journal of Engineering Science 49 (12), 1311-1325. 
Austin, R. A., McDowell, D. L., Jan. 2011. A dislocation-based constitutive model for viscoplastic deformation of fcc metals at very high strain rates. International Journal of Plasticity 27 (1), 1-24.

Austin, R. A., McDowell, D. L., May 2012. Parameterization of a rate-dependent model of shock-induced plasticity for copper, nickel, and aluminum. International Journal of Plasticity 32-33, 134-154.

Bammann, D. J., 2001. A model of crystal plasticity containing a natural length scale. Materials Science and Engineering: A 309, 406-410.

Bammann, D. J., Aifantis, E., 1982. On a proposal for a continuum with microstructure. Acta Mechanica $45(1-2), 91-121$.

Barton, N., Benson, D., Becker, R., 2005. Crystal level continuum modelling of phase transformations: the alpha-epsilon transformation in iron. Modelling and Simulation in Materials Science and Engineering 13 (5), 707-731.

Barton, N. R., Knap, J., Arsenlis, A., Becker, R., Hornung, R. D., Jefferson, D. R., 2008. Embedded polycrystal plasticity and adaptive sampling. International Journal of Plasticity 24 (2), 242-266.

Bayley, C. J., Brekelmans, W. A. M., Geers, M. G. D., Nov. 2006. A comparison of dislocation induced back stress formulations in strain gradient crystal plasticity. International Journal of Solids and Structures 43 (24), 7268-7286.

Becker, R., 2004. Effects of crystal plasticity on materials loaded at high pressures and strain rates. International Journal of Plasticity 20 (11), 1983-2006.

Bronkhorst, C., Hansen, B., Cerreta, E., Bingert, J., 2007. Modeling the microstructural evolution of metallic polycrystalline materials under localization conditions. Journal of the Mechanics and Physics of Solids 55 (11), 2351-2383.

Bronkhorst, C., Kalidindi, S., Anand, L., 1992. Polycrystalline plasticity and the evolution of crystallographic texture in fcc metals. Philosophical Transactions of the Royal Society of London. Series A: Physical and Engineering Sciences 341 (1662), 443-477.

Cao, F., Beyerlein, I. J., Addessio, F. L., Sencer, B. H., Trujillo, C. P., Cerreta, E. K., Gray, George T., I., 2010. Orientation dependence of shock-induced twinning and substructures in a copper bicrystal. Acta Materialia 58 (2), 549-559.

Cerreta, E. K., Escobedo, J. P., Perez-Bergquist, A., Koller, D. D., Trujillo, C. P., Gray, G. T., I., Brandl, C., Germann, T. C., 2012. Early stage dynamic damage and the role of grain boundary type. Scripta Materialia 66 (9), 638-641.

Clayton, J., 2013. Nonlinear eulerian thermoelasticity for anisotropic crystals. Journal of the Mechanics and Physics of Solids 61 (10), 1983 - 2014.

URL http://www.sciencedirect.com/science/article/pii/S0022509613001051

Clayton, J., McDowell, D., 2003. A multiscale multiplicative decomposition for elastoplasticity of polycrystals. International Journal of Plasticity 19 (9), 1401-1444.

Clayton, J. D., 2014. Analysis of shock compression of strong single crystals with logarithmic thermoelasticplastic theory. International Journal of Engineering Science 79, 1-20.

Clayton, J. D., McDowell, D. L., Bammann, D. J., 2004. A multiscale gradient theory for single crystalline elastoviscoplasticity. International journal of engineering science 42 (5), 427-457.

Cottrell, A. H., 1952. The formation of immobile dislocations during slip. Philosophical Magazine 43 (341), 645-647. 
Evers, L. P., Brekelmans, W. A. M., Geers, M. G. D., Oct. 2004a. Non-local crystal plasticity model with intrinsic ssd and gnd effects. Journal of the Mechanics and Physics of Solids 52 (10), 2379-2401.

Evers, L. P., Brekelmans, W. A. M., Geers, M. G. D., Sep. 2004b. Scale dependent crystal plasticity framework with dislocation density and grain boundary effects. International Journal of Solids and Structures 41 (18-19), 5209-5230.

Forest, S., 2009. Micromorphic approach for gradient elasticity, viscoplasticity, and damage. Journal of Engineering Mechanics 135 (3), 117-131.

Forest, S., Cailletaud, G., Sievert, R., 1997. A cosserat theory for elastoviscoplastic single crystals at finite deformation. Arch. Mech 49, 705-736.

Geers, M., Brekelmans, W., Bayley, C., 2007. Second-order crystal plasticity: internal stress effects and cyclic loading. Modelling and Simulation in Materials science and Engineering 15 (1), S133.

Gerken, J. M., Dawson, P. R., Apr. 2008. A crystal plasticity model that incorporates stresses and strains due to slip gradients. Journal of the Mechanics and Physics of Solids 56 (4), 1651-1672.

Girifalco, L., 2000. Statistical Mechanics of Solids. Oxford University Press.

Greeff, C., Graf, M., 2004. Lattice dynamics and the high-pressure equation of state of Au. Physical Review B 69 (5).

Greeff, C. W., Boettger, J. C., Graf, M. J., Johnson, J. D., 2006. Theoretical investigation of the Cu EOS standard. Journal of Physics and Chemistry of Solids 67 (9-10), 2033-2040.

Groma, I., Csikor, F. F., Zaiser, M., Mar. 2003. Spatial correlations and higher-order gradient terms in a continuum description of dislocation dynamics. Acta Materialia 51 (5), 1271-1281.

Gurtin, M. E., 2002. A gradient theory of single-crystal viscoplasticity that accounts for geometrically necessary dislocations. Journal of the Mechanics and Physics of Solids 50 (1), 5-32.

Gurtin, M. E., Anand, L., Lele, S. P., 2007. Gradient single-crystal plasticity with free energy dependent on dislocation densities. Journal of the Mechanics and Physics of Solids 55 (9), 1853-1878.

Han, C.-S., Gao, H., Huang, Y., Nix, W. D., 2005. Mechanism-based strain gradient crystal plasticity - i. theory. Journal of the Mechanics and Physics of Solids 53 (5), 1188-1203.

Han, W. Z., An, Q., Luo, S. N., Germann, T. C., Tonks, D. L., Goddard, W. A., I., 2012. Deformation and spallation of shocked $\mathrm{Cu}$ bicrystals with Sigma 3 coherent and symmetric incoherent twin boundaries. Physical Review B 85 (2), 024107.

Hartley, C. S., 2003. A method for linking thermally activated dislocation mechanisms of yielding with continuum plasticity theory. Philosophical Magazine 83 (31-34), 3783-3808.

Hirschberger, C. B., Peerlings, R. H. J., Brekelmans, W. A. M., Geers, M. G. D., Dec. 2011. On the role of dislocation conservation in single-slip crystal plasticity. Modelling and Simulation In Materials Science and Engineering 19 (8), 085002.

Hirth, J. P., Lothe, J., 1969. Stress fields of dislocation segments and forces on them. Strength and plasticity, Physics of, 39-46.

Hirth, J. P., Lothe, J., 1982. Theory of dislocations.

Hochrainer, T., Zaiser, M., Gumbsch, P., 2007. A three-dimensional continuum theory of dislocation systems: kinematics and mean-field formulation. Philosophical Magazine 87 (8-9), 1261-1282. 
Kalidindi, S. R., Bronkhorst, C. A., Anand, L., 1992. Crystallographic texture evolution in bulk deformation processing of fcc metals. Journal of the Mechanics and Physics of Solids 40 (3), 537-569.

Kocks, U. F., Argon, A. S., Ashby, M. F., 1975. Thermodynamics and kinetics of slip. Progress In Materials Science 19, 1-281.

Kröner, E., 1961. On the plastic deformation of polycrystals. Acta Metallurgica 9 (2), 155-161.

Kysar, J., Saito, Y., Oztop, M., Lee, D., Huh, W., 2010. Experimental lower bounds on geometrically necessary dislocation density. International Journal of Plasticity 26 (8), 1097-1123.

Lee, M., Lim, H., Adams, B., Hirth, J., Wagoner, R., 2010. A dislocation density-based single crystal constitutive equation. International Journal of Plasticity 26 (7), 925-938.

Leung, H., Leung, P., Cheng, B., Ngan, A., 2015. A new dislocation-density-function dynamics scheme for computational crystal plasticity by explicit consideration of dislocation elastic interactions. International Journal of Plasticity 67, 1-25.

Lim, H., Lee, M., Kim, J., Adams, B., Wagoner, R., 2011. Simulation of polycrystal deformation with grain and grain boundary effects. International Journal of Plasticity 27 (9), 1328-1354.

Limkumnerd, S., van der Giessen, E., May 2008a. Statistical approach to dislocation dynamics: From dislocation correlations to a multiple-slip continuum theory of plasticity. Physical Review B 77 (18), 184111.

Limkumnerd, S., van der Giessen, E., Nov. 2008b. Study of size effects in thin films by means of a crystal plasticity theory based on dift. Journal of the Mechanics and Physics of Solids 56 (11), 3304-3314.

Liu, Z., Zhuang, Z., Liu, X., Zhao, X., Zhang, Z., 2011. A dislocation dynamics based higher-order crystal plasticity model and applications on confined thin-film plasticity. International Journal of Plasticity 27 (2), $201-216$.

Lloyd, J. T., Clayton, J. D., Austin, R. A., McDowell, D. L., Sep. 2014. Plane wave simulation of elasticviscoplastic single crystals. Journal of the Mechanics and Physics of Solids 69, 14-32.

Luscher, D. J., Bronkhorst, C. A., Alleman, C. N., Addessio, F. L., Sep. 2013. A model for finite-deformation nonlinear thermomechanical response of single crystal copper under shock conditions. Journal of the Mechanics and Physics of Solids 61 (9), 1877-1894.

Ma, A., Roters, F., Raabe, D., 2006. A dislocation density based constitutive model for crystal plasticity fem including geometrically necessary dislocations. Acta Materialia 54 (8), 2169-2179.

Mathur, K. K., Dawson, P. R., 1989. On modeling the development of crystallographic texture in bulk forming processes. International Journal of Plasticity 5 (1), 67-94.

Mayeur, J. R., McDowell, D. L., Bammann, D. J., 2011. Dislocation-based micropolar single crystal plasticity: Comparison of multi-and single criterion theories. Journal of the Mechanics and Physics of Solids 59 (2), $398-422$.

Mayeur, J. R., Mourad, H. M., Luscher, D. J., Hunter, A., Kenamond, M. A., 2015. Numerical implementation of a continuum dislocation dynamics model of crystal plasticity, submitted.

Mourad, H. M., Mayeur, J. R., Luscher, D. J., Hunter, A., Kenamond, M. A., 2015. A dislocation-conserving model of crystal plasticity at high strain rates: Computational aspects and solution strategies, in preparation.

Nye, J. F., 1953. Some geometrical relations in dislocated crystals. Acta Metallurgica 1 (2), 153-162. 
Perez-Bergquist, A. G., Cerreta, E. K., Trujillo, C. P., Cao, F., Gray, G. T., I., 2011. Orientation dependence of void formation and substructure deformation in a spalled copper bicrystal. Scripta Materialia 65 (12), $1069-1072$.

Prasad, G., Goerdeler, M., Gottstein, G., 2005. Work hardening model based on multiple dislocation densities. Materials Science and Engineering: A 400, 231-233.

Reuber, C., Eisenlohr, P., Roters, F., Raabe, D., 2014. Dislocation density distribution around an indent in single-crystalline nickel: Comparing nonlocal crystal plasticity finite-element predictions with experiments. Acta Materialia 71, 333-348.

Roters, F., Raabe, D., Gottstein, G., 2000. Work hardening in heterogeneous alloys - a microstructural approach based on three internal state variables. Acta materialia 48 (17), 4181-4189.

Sandfeld, S., Hochrainer, T., Gumbsch, P., Zaiser, M., 2010. Numerical implementation of a 3d continuum theory of dislocation dynamics and application to micro-bending. Philosophical Magazine 90 (27-28), $3697-3728$.

Sandfeld, S., Hochrainer, T., Zaiser, M., Gumbsch, P., 2011. Continuum modeling of dislocation plasticity: Theory, numerical implementation, and validation by discrete dislocation simulations. Journal of Materials Research 26 (05), 623-632.

Schoenfeld, S. E., 1998. Dynamic behaviour of polycrystalline tantalum. International Journal of Plasticity $14(9), 871-890$.

Schulz, K., Dickel, D., Schmitt, S., Sandfeld, S., Weygand, D., Gumbsch, P., 2014. Analysis of dislocation pile-ups using a dislocation-based continuum theory. Modelling and Simulation in Materials Science and Engineering $22(2), 025008$.

Vinet, P., Ferrante, J., Smith, J., Rose, J., 1986. A universal equation of state for solids. Journal of Physics C - Solid State Physics 19 (20), L467-L473.

Vogler, T. J., Clayton, J. D., 2008. Heterogeneous deformation and spall of an extruded tungsten alloy: plate impact experiments and crystal plasticity modeling. Journal of the Mechanics and Physics of Solids 56 (2), $297-335$.

Walgraef, D., Aifantis, E. C., 1985a. On the formation and stability of dislocation patterns .1. one-dimensional considerations. International Journal of Engineering Science 23 (12), 1351-1358.

Walgraef, D., Aifantis, E. C., 1985b. On the formation and stability of dislocation patterns .2. twodimensional considerations. International Journal of Engineering Science 23 (12), 1359-1364.

Walgraef, D., Aifantis, E. C., 1985c. On the formation and stability of dislocation patterns .3. 3-dimensional considerations. International Journal of Engineering Science 23 (12), 1365-1372.

Wang, Z., Beyerlein, I., 2008. Stress orientation and relativistic effects on the separation of moving screw dislocations. Physical Review B 77 (18), 184112.

Winey, J. M., Gupta, Y. M., 2004. Nonlinear anisotropic description for shocked single crystals: Thermoelastic response and pure mode wave propagation. Journal of Applied Physics 96 (4), 1993-1999.

Winey, J. M., Gupta, Y. M., 2006. Nonlinear anisotropic description for the thermomechanical response of shocked single crystals: Inelastic deformation. Journal of Applied Physics 99 (2), 023510.

Yefimov, S., Groma, I., Van der Giessen, E., 2004. A comparison of a statistical-mechanics based plasticity model with discrete dislocation plasticity calculations. Journal of the Mechanics and Physics of Solids $52(2), 279-300$. 
Yefimov, S., van der Giessen, E., Jun. 2005a. Multiple slip in a strain-gradient plasticity model motivated by a statistical-mechanics description of dislocations. International Journal of Solids and Structures 42 (11-12), $3375-3394$.

Yefimov, S., van der Giessen, E., Mar. 2005b. Size effects in single crystal thin films: nonlocal crystal plasticity simulations. European Journal of Mechanics A-solids 24 (2), 183-193. 